\title{
Recent Advances in Understanding of Thermal Expansion Effects in Premixed Turbulent Flames
}

\author{
Vladimir A. Sabelnikov ${ }^{1}$ and Andrei N. Lipatnikov ${ }^{2}$ \\ ${ }^{1}$ ONERA, Palaiseau F-91761, France; email: vladimir.sabelnikov@onera.fr \\ ${ }^{2}$ Department of Applied Mechanics, Chalmers University of Technology, \\ Gothenburg SE-412 96, Sweden; email: lipatn@chalmers.se
}

\section{Keywords}

premixed turbulent combustion, countergradient transport, flame-generated turbulence, Darrieus-Landau instability, turbulent burning velocity, mean flame brush thickness

\begin{abstract}
When a premixed flame propagates in a turbulent flow, not only does turbulence affect the burning rate (e.g., by wrinkling the flame and increasing its surface area), but also the heat release in the flame perturbs the pressure field, and these pressure perturbations affect the turbulent flow and scalar transport. For instance, the latter effects manifest themselves in the so-called countergradient turbulent scalar flux, which has been documented in various flames and has challenged the combustion community for approximately 35 years. Over the past decade, substantial progress has been made in investigating (a) the influence of thermal expansion in a premixed flame on the turbulent flow and turbulent scalar transport within the flame brush, as well as $(b)$ the feedback influence of countergradient scalar transport on the turbulent burning rate. The present article reviews recent developments in this field and outlines issues to be solved in future research.
\end{abstract}




\section{INTRODUCTION}

Premixed turbulent combustion (i.e., the burning of a homogeneous mixture of fuel and air) is widely used for energy conversion in land transportation (e.g., piston engines in cars), aviation (e.g., aero-engine afterburners), and stationary power generation (e.g., gas engines and turbines). Accordingly, properly responding to environmental challenges such as the threat of global warming and securing sustainable development of the postindustrial society require finding and implementing new innovative technological solutions capable of satisfying stringent requirements for ultraclean, fuel-flexible, and highly efficient future internal combustion engines for energy conversion in stationary power plants and vehicles. For the development of such solutions, advanced research tools are needed, in particular, high-fidelity predictive models that are based on deep understanding of the governing physical mechanisms of flame-turbulence interaction and that adequately respond to new challenges associated with, for example, emission reduction, new burning modes, and alternative fuels. However, despite long-term usage of and research into premixed turbulent combustion, contemporary physical and numerical models of flame-turbulence interaction are still limited in their ability to predict basic flame characteristics quantitatively and even qualitatively. Major difficulties in understanding premixed turbulent combustion are associated with turbulent burning being a highly nonlinear and multiscale phenomenon, which locally involves various processes, such as chemical reactions, heat release and thermal expansion, molecular transport, and turbulence. Accordingly, various local phenomena can occur in a premixed flame.

More specifically, from a global perspective, the propagation of a premixed flame can occur in two regimes, and in each regime, two types of effects require consideration. If unburned gas flow is laminar, then $(a)$ the flow nonuniformities affect the flame and, in particular, change its local speed, as first pointed out by Markstein (1951), while (b) combustion-induced thermal expansion affects the flow by generating pressure perturbations, which can cause hydrodynamic flame instability, predicted theoretically by Darrieus (1938) and Landau (1944). Similarly, if unburned gas flow is turbulent, then $(c)$ the turbulence affects the flame and can significantly accelerate its propagation by increasing the flame-surface area, as first modeled by Damköhler (1940) and Shchelkin [1967 (1943)], while $(d)$ pressure perturbations due to combustion-induced thermal expansion affect the turbulent flow, with flame-generated turbulence hypothesized (Karlovitz et al. 1951, Scurlock \& Grover 1953) to significantly increase the burning rate.

Although each of the effects $(a)-(d)$ was studied over more than six decades ago, the levels of understanding of the different effects are significantly different. The greatest progress has been made in investigating the interaction between a premixed flame and a laminar flow [i.e., effects $(a)$ and $(b)$ ]. However, results obtained by investigating laminar flames cannot be applied straightforwardly to the description of burning in internal combustion engines, which is typically turbulent. Nevertheless, such results are a cornerstone for fundamental understanding of flame-turbulence interaction. Accordingly, they are addressed in the present review, and the reader interested in a more detailed discussion of laminar flame theory is referred to reviews previously published in this journal (Sivashinsky 1983, Buckmaster 1993, Clavin 1994, Matalon 2007).

The effects of turbulence on premixed flames [i.e., effects $(c)$ ] were the focus of combustion research for decades (Chomiak 1990, Kuznetsov \& Sabelnikov 1990, Peters 2000, Poinsot \& Veynante 2005, Echekki \& Mastorakos 2011, Swaminathan \& Bray 2011, Lipatnikov 2012). These studies have shown that, in a wide range of conditions typical for internal combustion engines, the turbulent burning rate is mainly controlled by turbulence characteristics; for example, the speed of propagation of a flame against a turbulent flow of premixed reactants is on the order of the 
root-mean-square (rms) turbulent velocity $u^{\prime}$. Accordingly, for a physical or numerical model to be able to predict the turbulent burning rate, the effects of thermal expansion on turbulent flow [i.e., effects $(d)$ ] should be well understood and properly taken into account.

Contrary to the three other aforementioned effects, developments in this area were rather moderate and rarely reviewed (Günther 1983, Bray 1995, Lipatnikov \& Chomiak 2010). Over the past decade, there has been substantial progress in investigating thermal expansion effects in premixed turbulent flames, and encouraging results have been obtained. Although a wellrecognized comprehensive theory has not yet resulted, some models have been developed and some local phenomena have been newly revealed. Recent progress in understanding of thermal expansion effects on turbulent flow offers an opportunity to advance the earlier state of the art and substantially improve the combustion community's capabilities for quantitatively predicting the turbulent burning rate and other important flame characteristics, such as its thickness, mean structure, and emissions from flames. The goal of the present article is to discuss these recent developments and to emphasize unresolved issues.

Sections 3 and 4 review the state-of-the-art research into the effects of thermal expansion in premixed flames on turbulent flow and scalar transport, respectively. However, before considering these basic issues, we briefly introduce the reader to the physical and mathematical background required to follow the subsequent discussion.

\section{DISCUSSION FRAMEWORK}

Depending on the Reynolds number and ratio $u^{\prime} / S_{\mathrm{L}}$ of the rms turbulent velocity to the laminar flame speed, premixed turbulent combustion occurs in various regimes (Borghi 1988, Peters 2000), ranging from chemical reactions localized to thin flamelets in large-scale (when compared to the laminar flame thickness $\delta_{\mathrm{L}}$ ) turbulence [Damköhler 1940, Shchelkin 1967 (1943)] to distributed burning in small-scale turbulence (Damköhler 1940). The focus of the present article is placed on the former (i.e., flamelet) regime because the response of a turbulent flow to localized thermal expansion is an issue specific to premixed combustion and is associated with stronger effects when compared to distributed thermal expansion.

Solely combustion-induced thermal expansion is addressed, whereas dynamic compressibility effects (e.g., the interaction of flames with acoustic and shock waves) are beyond the scope of the present article. Accordingly, the flow of unburned or burned gas is considered to be incompressible. A complete set of equations that model such low-Mach number premixed combustion can be found elsewhere (e.g., Majda \& Sethian 1985). In the following, the term thermal expansion refers to thermal expansion due to heat release in a flame front.

There are two widely used approaches to modeling premixed turbulent combustion in the flamelet regime. If the internal structure of thin flamelets is disregarded, then unburned and burned mixtures are considered to be separated by an interface (infinitely thin flame front) that propagates at a speed $S_{\mathrm{L}}$ into the unburned gas. Similar to a laminar premixed flame (Zel'dovich et al. 1985), the density and pressure drop from $\rho_{\mathrm{u}}$ to $\rho_{\mathrm{b}}$ and from $p_{\mathrm{u}}$ to $p_{\mathrm{b}}=p_{\mathrm{u}}-\rho_{\mathrm{u}} \tau S_{\mathrm{L}}^{2}$, respectively, at the front, whereas the local normal (to the front) flow velocity increases [i.e., $\left.\left|\left(\mathbf{u}_{\mathrm{b}}-\mathbf{u}_{\mathrm{u}}\right) \cdot \mathbf{n}\right|=\tau S_{\mathrm{L}}\right]$. Here, $\mathbf{n}$ is the local unity normal vector pointing to unburned gas; $\tau=\sigma-1$ is the heat release factor; the subscripts $\mathrm{b}$ and $\mathrm{u}$ refer to burned and unburned gas, respectively; and $\sigma=\rho_{\mathrm{u}} / \rho_{\mathrm{b}}$ is the density ratio, which varies from 5 to 7.5 in typical hydrocarbon-air flames under room-temperature conditions.

Accordingly, $S_{\mathrm{L}}$ and $\sigma$ are considered to be the major mixture characteristics. They are controlled by the fuel formula, equivalence ratio $\Phi$, unburned gas temperature $T_{\mathrm{u}}$, and pressure $p$. Because $\left|p_{\mathrm{u}}-p_{\mathrm{b}}\right| \ll p$ in a typical flame, local pressure variations are neglected when evaluating
Laminar flame speed

$\left(\mathrm{S}_{\mathrm{L}}\right)$ : basic

characteristic of a

combustible mixture

(fuel structure,

stoichiometry,

pressure, and

temperature); i.e., the speed of a planar 1D flame that propagates into a homogeneous quiescent mixture of fuel and oxidant

Flamelet: a thin (when compared to local scales of upstream flow inhomogeneities) inherently laminar zone that separates the upstream unburned mixture and downstream combustion products

Equivalence ratio $(\Phi)$ : a ratio of the fuel mass fraction in a mixture to the stoichiometric fuel mass fraction required for the fuel and oxygen to be fully burned 
BML approach: approach to modeling premixed turbulent combustion developed by Bray, Moss, and Libby by assuming that variations in mixture characteristics are localized to flamelets
$S_{\mathrm{L}}$ or $\sigma$ but control $\left(\mathbf{u}_{\mathrm{b}}-\mathbf{u}_{\mathrm{u}}\right) \cdot \mathbf{n}$. Therefore, the local pressure drop is of great importance when considering the effects of thermal expansion on turbulent flow and transport.

Let us apply a two-fluid model by Kataoka (1986) to the considered problem and introduce (a) a continuous function $G(\mathbf{x}, t)$ such that $G<0$ in unburned gas, $G=0$ at the flame front, and $G>0$ in combustion products; $(b)$ an indicator function $c(\mathbf{x}, t)=H[G(\mathbf{x}, t)]$, where $H$ is the Heaviside function, i.e., $c=0$ and 1 in the unburned and burned mixtures, respectively; and $(c)$ a unit normal vector $\mathbf{n}=-(\nabla G /|\nabla G|)_{G=0}$. Then, for a scalar, vector, or tensor quantity $q$ that is bounded at the flame front, $q=(1-c) q_{\mathrm{u}}+c q_{\mathrm{b}}$ and its mean value $\bar{q}$ can be evaluated as follows:

$$
\bar{q}(\mathbf{x}, t)=[1-\bar{c}(\mathbf{x}, t)] \bar{q}_{\mathrm{u}}(\mathbf{x}, t)+\bar{c}(\mathbf{x}, t) \bar{q}_{\mathrm{b}}(\mathbf{x}, t),
$$

where $\bar{c}(\mathbf{x}, t)$ is the probability of finding the burned mixture and values of $q$ conditioned on the unburned and burned gases; that is, $\bar{q}_{\mathrm{u}}(\mathbf{x}, t)$ and $\bar{q}_{\mathrm{b}}(\mathbf{x}, t)$, respectively, are defined as follows:

$$
[1-\bar{c}(\mathbf{x}, t)] \bar{q}_{\mathrm{u}}(\mathbf{x}, t) \equiv \overline{(1-c) q_{\mathrm{u}}}(\mathbf{x}, t), \quad \bar{c}(\mathbf{x}, t) \bar{q}_{\mathrm{b}}(\mathbf{x}, t) \equiv \overline{c q_{\mathrm{b}}}(\mathbf{x}, t) .
$$

Equation 1 was introduced into combustion theory by Prudnikov (1960). Readers interested in taking conditional averages of derivatives of discontinuous quantities (e.g., $\nabla p$ or dilatation $\nabla \cdot \mathbf{u}$ at a flame front) are referred to Kataoka (1986) given that such conditioned derivatives are not used in the present article. In the following, the dependencies of $c, \bar{q}_{\mathrm{u}}, \bar{q}_{\mathrm{b}}$, etc. on the spatial coordinates $\mathbf{x}$ and time $t$ are not specified, and mixture characteristics such as density $\rho_{\mathrm{u}}\left(\rho_{\mathrm{b}}\right)$ and temperature $T_{\mathrm{u}}\left(T_{\mathrm{b}}\right)$ of unburned (burned) gas are considered as constant input parameters.

Substitution of $q=\rho \mathbf{u}, q=\rho c, q=\rho \mathbf{u} c$, and $q=\rho u_{i} u_{j}$ into Equation 1 results in

$$
\begin{aligned}
& \tilde{\mathbf{u}}=(1-\tilde{c}) \overline{\mathbf{u}}_{\mathrm{u}}+\tilde{c} \overline{\mathbf{u}}_{\mathrm{b}}, \quad \overline{\rho \mathbf{u}^{\prime \prime} c^{\prime \prime}}=\overline{\rho \mathbf{u} c}-\bar{\rho} \tilde{\mathbf{u}} \tilde{c}=\bar{\rho} \tilde{c}(1-\tilde{c})\left(\overline{\mathbf{u}}_{\mathrm{b}}-\overline{\mathbf{u}}_{\mathrm{u}}\right), \\
& \overline{\rho u_{i}^{\prime \prime} u_{j}^{\prime \prime}}=\bar{\rho}\left[(1-\tilde{c})\left(\overline{u_{i}^{\prime} u_{j}^{\prime}}\right)_{\mathrm{u}}+\tilde{c}\left(\overline{u_{i}^{\prime} u_{j}^{\prime}}\right)_{\mathrm{b}}+\tilde{c}(1-\tilde{c})\left(\bar{u}_{i, \mathrm{~b}}-\bar{u}_{i, \mathrm{u}}\right)\left(\bar{u}_{j, \mathrm{~b}}-\bar{u}_{j, u}\right)\right],
\end{aligned}
$$

where $\left(\overline{u_{i}^{\prime} u_{j}^{\prime}}\right)_{\mathrm{u}}=\left[\overline{\left(u_{i}-\bar{u}_{i, \mathrm{u}}\right)\left(u_{j}-\bar{u}_{j, \mathrm{u}}\right)}\right]_{\mathrm{u}}$ and $\left(\overline{u_{i}^{\prime} u_{j}^{\prime}}\right)_{\mathrm{b}}=\left[\overline{\left(u_{i}-\bar{u}_{i, \mathrm{~b}}\right)\left(u_{j}-\bar{u}_{j, \mathrm{~b}}\right)}\right]_{\mathrm{b}}$ are correlations conditioned on unburned and burned gas, respectively; $\tilde{q} \equiv \overline{\rho q} / \bar{\rho}$ is the Favre (mass-weighted) average of $q$ and $q^{\prime \prime} \equiv q-\tilde{q}$. The combustion literature uses Favre-averaged quantities (Bray \& Libby 1976) because the Favre-averaged transport equations have a more compact form when compared to the counterpart Reynolds-averaged equations, which involve extra unclosed terms such as $\overline{\rho^{\prime} \mathbf{u}^{\prime}}$, where $\mathbf{u}^{\prime}=\mathbf{u}-\overline{\mathbf{u}}$.

If $q$ is equal to the mass fraction $Y$ of the deficient reactant, then Equation 1 yields $\bar{Y}=Y_{\mathrm{u}}(1-\bar{c})$ because $Y_{\mathrm{b}}=0$. Therefore, $c$ can be associated with the normalized reactant mass fraction (i.e., $\left.c=1-Y / Y_{\mathrm{u}}\right)$, and the following Favre-averaged transport equation

$$
\frac{\partial}{\partial t}(\bar{\rho} \tilde{c})+\nabla \cdot(\bar{\rho} \tilde{\mathbf{u}} \tilde{c})=-\nabla \cdot \overline{\rho \mathbf{u}^{\prime \prime} c^{\prime \prime}}+\bar{W}
$$

results straightforwardly from a transport equation for $Y / Y_{\mathrm{u}}$. Here, one obtains $\bar{W} \equiv \overline{S_{\mathrm{L}}|\nabla c|}$.

Another approach to modeling premixed turbulent burning in the flamelet regime was developed by Bray \& Libby (1976) and Bray \& Moss (1977), referred to as the BML approach. It allows for a finite flamelet thickness, considers $\delta_{\mathrm{L}}$ to be an important mixture characteristic in addition to $S_{\mathrm{L}}$ and $\sigma$, and offers wider opportunities to model effects of turbulence on combustion. Nevertheless, the two approaches (an infinitely thin flame front or a flamelet of finite thickness) are almost equally useful when modeling major effects of thermal expansion on turbulent flow. Within the framework of the BML approach, $c=1-Y / Y_{\mathrm{u}}$ is a combustion progress variable, $\mathbf{n}=-\nabla c /|\nabla c|$ is defined within flamelets, Equations $2-5$ hold to leading order with respect to a ratio $\delta_{\mathrm{L}} / \delta_{t} \ll 1$ of the laminar and turbulent flame brush thicknesses, and $\bar{W}=\nabla \cdot \overline{(\rho D \nabla c)}+\bar{w}_{c}$, where $\delta_{t}=1 / \max |\nabla \bar{c}|$ is the mean turbulent flame brush thickness, $D$ is the molecular diffusivity 
of the deficient reactant and $w_{c}$ is its consumption rate divided by $Y_{\mathrm{u}}$. With the exception of Section 3.3, below we consider infinitely thin flame fronts if the opposite is not stated.

Within the Reynolds-averaged Navier-Stokes (RANS) framework, the two main challenges of premixed turbulent combustion modeling consist of closing the turbulent scalar flux $\overline{\rho \mathbf{u}^{\prime \prime} c^{\prime \prime}}$ and closing the source term $\bar{W}$. In the present article, we address the problem of modeling the flux and turbulence characteristics required by a closure relation for $\bar{W}$. Models that yield a closure relation for $\bar{W}$ (Chomiak 1990, Kuznetsov \& Sabelnikov 1990, Peters 2000, Poinsot \& Veynante 2005, Echekki \& Mastorakos 2011, Swaminathan \& Bray 2011, Lipatnikov 2012) focus on the influence of turbulence on combustion but rarely address the influence of thermal expansion on turbulence. Moreover, we restrict ourselves to the RANS framework because largeeddy simulation (LES) models of the flame-turbulence interaction are strongly based on their RANS counterparts. Readers interested in LES of turbulent combustion are referred to the review by Pitsch (2006).

In the following, Equations 2-5 are often applied to a statistically unperturbed flame (SUF) and impinging jet flame (IJF) that are described by statistically one-dimensional (1D) transport equations. In such cases, we use $1 \mathrm{D}$ Equations $2-5$, where $u$ is the $x$ component of the flow velocity vector, which is normal to the mean flame brush.

Finally, it is worth introducing the following basic characteristics of premixed turbulent flames (e.g., Driscoll 2008, Lipatnikov 2012). The burning (consumption) velocity $U_{t}$ is equal to $\rho_{\mathrm{u}}^{-1} \int_{-\infty}^{\infty} \bar{W} \mathrm{~d} x$ in an SUF or IJF. The flame (displacement) speed $S_{t}$ is equal to $U_{t}$ in a fully developed SUF but depends on the choice of a mean flame surface $\bar{c}(x, t)=$ const in an IJF or developing SUF. The mean flame brush thickness $\delta_{t}$ is equal to $1 / \max \{|\nabla \bar{c}|\}$. The mean pressure $\operatorname{drop} \delta \bar{p}$ at the flame is typically much less than $p$ and is equal to $\rho_{\mathrm{u}} \tau U_{t}^{2}$ in a fully developed SUF.

\section{INFLUENCE OF THERMAL EXPANSION ON TURBULENT FLOW}

\subsection{Flame-Generated Turbulence?}

The problem of flame-generated turbulence was posed by Karlovitz et al. (1951) and Scurlock \& Grover (1953) in order to attribute high values of $S_{t}$ measured in early experiments to an increase in $u^{\prime}$ within flame brushes when compared to $u^{\prime}$ in upstream flows. Karlovitz et al. (1951) argued that, because of the random orientation of flame fronts, the local velocity jumps $\left|\left(\mathbf{u}_{\mathrm{b}}-\mathbf{u}_{\mathrm{u}}\right) \cdot \mathbf{n}\right|$ increase the magnitude $u^{\prime}=\overline{|\mathbf{u}-\overline{\mathbf{u}}|} / \sqrt{3}$ of velocity fluctuations in products. In other words, Karlovitz et al. (1951) associated flame-generated turbulence with flow acceleration by the local pressure drop $\delta p_{\mathrm{f}}$ at flame fronts.

Scurlock \& Grover (1953) highlighted (a) the creation of transverse shear in the flow of burned products within a flame brush due to uneven axial flow acceleration by the mean pressure gradient $\nabla \bar{p}$ and $(b)$ turbulence generation by the shear flow. Because the acceleration of a fluid particle $D \mathbf{u} / D t \propto \rho^{-1} \nabla p$ is inversely proportional to its density, the same pressure gradient accelerates low-density products more strongly than heavier unburned gas. Outside the front within the flame brush, $\nabla p$ is induced because of thermal expansion in surrounding pieces of the front. Accordingly, because of the preferential product acceleration by $\nabla p$, the axial velocity $u\left(x, y_{1}, z_{1}\right)$ of a product particle that has passed the front at a larger axial distance $\delta x_{1}\left(y_{1}, z_{1}\right)$ from a plane $x=$ const, which is parallel to the flame brush, is expected to be larger than the velocity $u\left(x, y_{2}, z_{2}\right)$ of a neighboring product particle that has passed the front at a shorter axial distance $\delta x_{2}\left(y_{2}, z_{2}\right)$ from the same plane.

As reviewed elsewhere (Günther 1983, Lipatnikov \& Chomiak 2010) and shown in Figure 1, various experimental and direct numerical simulation (DNS) studies of weakly turbulent flames associated with the flamelet combustion regime documented an increase in Reynolds- or
Reynolds-averaged Navier-Stokes

(RANS) equations: describe large-scale spatial and temporal variations of the mean flow velocity, but require a model of the influence of turbulent fluctuations on these variations

Large-eddy simulation (LES): simulation technique that deals with transport equations averaged over a small spatial volume using a low-pass filter; the LES equations resolve turbulent eddies larger than the filter width

Statistically unperturbed flame (SUF): statistically planar 1D turbulent premixed flame

Impinging jet flame (IJF): open flame stabilized upstream of a plate placed perpendicularly to a turbulent jet of a fuel-air mixture at a certain distance from the nozzle

Fully developed premixed turbulent flame: flame that propagates at a statistically stationary speed and has a statistically stationary thickness

Direct numerical simulation (DNS): solution of transport equations using a numerical grid that is sufficiently fine to resolve the smallest turbulent eddies and mixture nonuniformities 

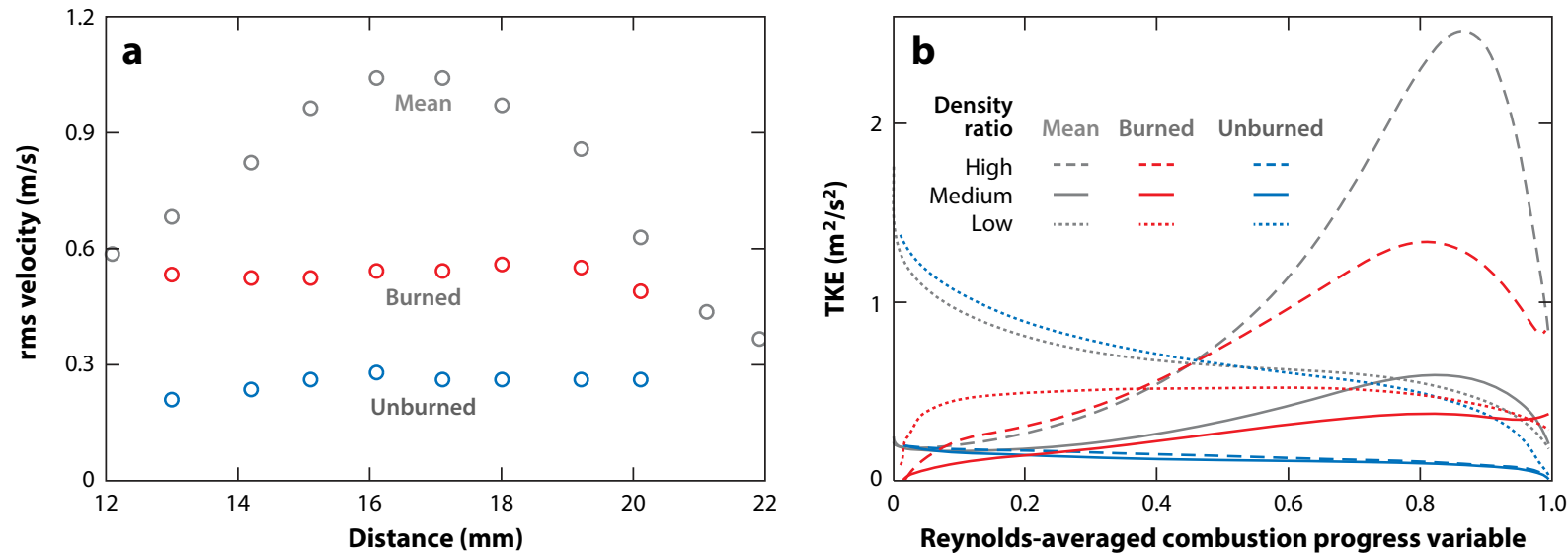

Figure 1

(a) Mean and conditioned root-mean-square velocities normal to a mean flame brush (from 13 to $20 \mathrm{~mm}$ ), measured by Cheng \& Shepherd (1986), along a normal to an open oblique premixed turbulent flame (products on the left). (b) Mean and conditioned turbulent kinetic energy (TKE) extracted from direct numerical simulation data by Nishiki et al. (2002) obtained from a statistically unperturbed flames (product on the right) characterized by $\sigma=7.53$ (high density ratio), 5.0 (medium density ratio), and 2.5 (low density ratio). With a low density ratio, the plotted TKE is increased by a factor of 10 .

TKE: turbulent kinetic energy

Lewis number $(L e)$ : a ratio of the molecular heat diffusivity of a combustible mixture to the molecular mass diffusivity of the deficient reactant in the mixture
Favre-averaged turbulent kinetic energy (TKE), $\bar{k}=\overline{(\mathbf{u}-\overline{\mathbf{u}})^{2}} / 2$ or $\tilde{k}=\overline{\rho(\mathbf{u}-\tilde{\mathbf{u}})^{2}} / 2 \bar{\rho}$, respectively, and in $u^{\prime}$ that characterizes the velocity vector (e.g., $u^{\prime}=\sqrt{2 \bar{k} / 3}$ or $u^{\prime}=\sqrt{2 \tilde{k} / 3}$ ) or its single component [e.g., $u_{j}^{\prime 2}=\overline{\left(u_{j}-\bar{u}_{j}\right)^{2}}$ ], with the effect mitigated by an increase in $u^{\prime} / S_{\mathrm{L}}$.

The mainstream approach to RANS simulations of flame-generated turbulence in premixed flames, pioneered by Bray \& Libby (1976), Librovich \& Lisitzyn (1977), and Libby \& Bray (1977), consists of physically and/or numerically (DNS) modeling various terms in a transport equation for $\tilde{k}$ or $\bar{k}$. The approach was mainly developed in the 1980s and 1990s, with the results critically discussed recently (Lipatnikov \& Chomiak 2010, section 4.1). Briefly, various closed equations that involved a set of model constants were proposed, but none was validated against a wide set of substantially different experimental or DNS data by retaining all the constants unchanged. Moreover, if a closure relation obtained by a research group was subsequently tested by other experts by processing other DNS data, there were always data that contradicted this closure relation at least quantitatively. For instance, recent DNS by Chakraborty et al. (2011) shows that terms in the $\tilde{k}$ transport equation are sensitive to the Lewis number, whereas earlier models of flame-generated turbulence did not allow for $L e$.

A few years ago, Kolla et al. (2014) defined a new density-weighted two-point velocity correlation and derived a balance equation for the counterpart spectrum function in wave-number space. An analysis of this equation has shown, in particular, that "pressure-dilatation correlation is a source of kinetic energy at high wave numbers" (Kolla et al. 2014, p. 456). It is worth remembering that, in the RANS context, such a correlation $\overline{p^{\prime} \nabla \cdot \mathbf{u}^{\prime}}$ was first highlighted by Kuznetsov (1979), and an important role played by this term in the transport equation for $\tilde{k}$ was subsequently supported by DNS data, as reviewed elsewhere (Lipatnikov \& Chomiak 2010). Kolla et al. (2014) analyzed other DNS data, which were not associated with the flamelet combustion regime, and found that the pressure-dilatation correlation in the new spectrum equation caused an inflection of the spectrum drop-off at large wave numbers. Lipatnikov et al. (2015a) reported the influence of thermal expansion on velocity spectra conditioned to unburned gas at low $\bar{c}$ in DNS of SUFs associated with the flamelet combustion regime. Earlier, Furukawa et al. (2002) compared spectra 
conditioned to unburned and burned mixtures, measured in the middle of a flame brush, and found that large- and small-scale velocity fluctuations were more intense in the burned and unburned gases, respectively.

As far as an effect of intensification of velocity fluctuations behind a flame front on the burning rate and $S_{t}$ is concerned, two fundamental issues have not yet been resolved. First, because a flame front propagates into unburned gas, perturbations of the upstream flow of the unburned gas are required to accelerate the flame propagation. This issue was already recognized by Karlovitz et al. (1951, p. 546), who hypothesized that turbulence generated behind a flame front diffused "against the flow before the flame front." However, since that pioneering study, we have not substantially advanced our understanding of the magnitude and physical mechanisms of the effects of velocity fluctuations in combustion products on the upstream flow of unburned gas and turbulent flame speed. We further discuss the influence of thermal expansion on the upstream flow of unburned gas in Section 3.4.

Second, the use of TKE and rms velocity determined in a standard manner (e.g., $\tilde{k}=$ $\overline{\rho(\mathbf{u}-\tilde{\mathbf{u}})^{2}} / 2 \bar{\rho}$ and $\left.u^{\prime}=\sqrt{2 \tilde{k} / 3}\right)$ for characterizing turbulence appears to be fundamentally flawed if combustion occurs in the flamelet regime. Indeed, Equation 4 clearly shows that the second moments of the velocity field are straightforwardly affected by the difference in velocities conditioned to burned and unburned mixtures. If this difference is on the order of $\tau S_{\mathrm{L}}$, then the last term on the right-hand side of Equation 4 scales as $\left(\tau S_{\mathrm{L}}\right)^{2}$ and can be much larger than the two other terms in the case of weak turbulence [i.e., $u^{\prime} / S_{\mathrm{L}}=O(1)$ ]. However, $(a)$ a local normal velocity jump due to thermal expansion at a flame front and $(b)$ turbulence considered to be inherently rotational $3 \mathrm{D}$ flow appear to be two fundamentally different phenomena, which should be characterized by different quantities.

Accordingly, conditioned Reynolds stresses, TKE, and rms velocities are often assumed to properly characterize turbulence within a premixed flame brush. As reviewed elsewhere (Lipatnikov \& Chomiak 2010, section 3.2.3), conditioned second moments of the velocity field were evaluated in various experimental and DNS studies of premixed flames, which showed that such moments are less affected by combustion than the counterpart unconditioned moments (e.g., see Figure 1).

Figure 1 also indicates that the second moments conditioned to products are larger than the second moments conditioned to unburned gas in three flames, whereas the trend is opposite in the DNS case characterized by a low density ratio. As reviewed by Lipatnikov \& Chomiak (2010), various relations between $\bar{k}_{\mathrm{u}}$ and $\bar{k}_{\mathrm{b}}$ or $u_{\mathrm{u}}^{\prime}$ and $u_{\mathrm{b}}^{\prime}$ were documented in various flames. A model has not yet been developed that can predict the behavior of the conditioned second moments of the velocity field in the flamelet regime of premixed turbulent combustion. Balance equations for the conditioned Reynolds stresses and TKE in premixed flames were derived by Chen et al. (1986), Im et al. (2004), and Lipatnikov (2008) using different methods, but these equations involve a number of unclosed terms, and the proposed models of these terms require validation.

Moreover, the characterization of turbulence within a premixed flame brush with conditioned second moments of $\mathbf{u}(\mathbf{x}, t)$ can be disputed. Because of the random motion of an interface that separates two fluids, a statistical subensemble over which a conditional average is taken depends on $\mathbf{x}$ and $t$, as well known in the theory of intermittent flows (Libby 1975, Townsend 1976, Kuznetsov \& Sabelnikov 1990). As a result, the conditioned second moments differ from their mean counterparts, even in the case of the self-propagation of a passive interface in a constantdensity flow. For combustion applications, this feature of conditionally averaged second moments follows from Equations 3 and 4, was demonstrated by analyzing simple model problems (Lipatnikov 2009, 2011a), and was recently shown in a 3D DNS study of self-propagating interfaces in constant-density turbulent flows (Yu et al. 2014, 2015b). The DNS also indicated 
that quantities controlled by velocity gradients were significantly less sensitive to the averaging method. For instance, the conditioned and mean enstrophies $\omega^{2}=(\nabla \times \mathbf{u})^{2}$ or total strains $S^{2}$ were almost equal to one another in all simulated cases, thus implying that, e.g., $\left(\overline{S^{2}}\right)_{\mathrm{u}}$ is a proper characteristic of turbulence in reactants, at least in the case of constant density. Here, the total strain $S^{2}=S_{i j} S_{i j}$ is the square of the norm of the rate-of-strain tensor $S_{i j}=\left(\partial u_{i} / \partial x_{j}+\partial u_{j} / \partial x_{i}\right) / 2$, and the summation convention applies for the repeated indexes $i$ and $j$. These DNS data call for target-directed research into the behavior of $\nabla \times \mathbf{u}, \omega^{2}$, and $S^{2}$ in premixed turbulent flames.

\subsection{Vorticity, Enstrophy, and Total Strain}

The influence of turbulence on premixed combustion in the flamelet regime is commonly associated with both an increase in the flamelet surface and local variations in the burning rate per unit flamelet surface area, with both effects caused by turbulent stretching (e.g., Lipatnikov 2012). The stretch rate of the flamelet surface is not straightforwardly affected by the local vorticity but is controlled by the flamelet curvature and local strain rate (e.g., Matalon 2007). Nevertheless, the strain rate and vorticity are indissoluble in turbulent flow, as follows from their transport equations (Tsinober 2009).

In nonreacting flows, research for many years focused on coupling between $\nabla \times \mathbf{u}, S_{i j}, \omega^{2}$, and $S^{2}$ (e.g., Tsinober 2009). In contrast, we are aware of few studies of the behavior of $S^{2}$ and its coupling with the vorticity field in premixed turbulent combustion. Steinberg \& Driscoll (2009) measured 3D velocity gradients in premixed turbulent flames and found that flamelet stretching was caused by coherent strain-rate structures, which attenuated quickly behind the flamelets due to thermal expansion and viscous dissipation. The data by Steinberg \& Driscoll (2009, figure 24b) also indicate that the effects of turbulent eddies on the flame-surface area are better characterized by the total strain when compared to enstrophy or $u^{\prime}$.

Hamlington et al. (2011) performed DNS of SUFs in small-scale turbulence and reported the attenuation of $S^{2}$ due to combustion at $u^{\prime} / S_{\mathrm{L}}=2.45$, but a weak effect of the flames on $S^{2}$ at $u^{\prime} / S_{\mathrm{L}}>9.8$ (those flames are not associated with the flamelet combustion regime). Experiments by Steinberg et al. $\left(2015\right.$, p. 1287) indicated the attenuation of $S^{2}$ and $\omega^{2}$ at $u^{\prime} / S_{\mathrm{L}}=4.25$ but did not reveal any change "in the relative alignment of vorticity and strain rate" in a premixed jet flame.

The effects of premixed flames on the vorticity and enstrophy have received more attention. Averaging of vorticity and enstrophy transport equations yields several unclosed terms (e.g., Lipatnikov \& Chomiak 2010), with, e.g., baroclinic torque $\overline{\rho^{-2} \nabla \rho \times \nabla p}$ and dilatation $\overline{(\nabla \cdot \mathbf{u})(\nabla \times \mathbf{u})}$ terms in the vorticity equation being specific to combustion. Moreover, an increase in $T$ results in an increase in the kinematic viscosity $v$ and the magnitude of the viscous dissipation term. Although closure relations for such terms have not yet been elaborated, the effects of these terms on the vorticity and enstrophy in premixed flames were addressed in recent DNS studies of SUFs. These studies have shown that in the flamelet combustion regime, and if $\sigma \geq 4$, dilatation tends to reduce vorticity, but the anisotropic generation of vorticity occurs at flame fronts due to baroclinic torque, which overwhelms the dilatation and weak viscous dissipation, with the vorticity generation mitigated by a decrease in the density ratio (Treurniet et al. 2006, Lipatnikov et al. 2014). Moreover, if $\sigma \leq 2.5$, enstrophy attenuates within a flame brush due to viscous dissipation (Treurniet et al. 2006, Lipatnikov et al. 2014). Additionally, in small-scale intense turbulence, baroclinic torque and dilatation play a less important role when compared to vorticity generation due to vortex stretching and viscous dissipation, and vorticity attenuates within flames (Hamlington et al. 2011, Chakraborty et al. 2016), with the influence of combustion 
on the vorticity field mitigated by $u^{\prime} / S_{\mathrm{L}}$ (Hamlington et al. 2011). Finally, in small-scale, highly intense turbulence, the influence of combustion on the vorticity field is reduced to an increase in $v$ in hot products (Bobbitt et al. 2016).

Even in sufficiently intense turbulence, a decrease in the Lewis number results in vorticity generation due to a significant increase in the local baroclinic torque (Chakraborty 2014, Chakraborty et al. 2016) because faster (slower) diffusion of reactants (heat) into (from) thin, inherently laminar reaction zones stretched by turbulent eddies leads to a local increase in the burning rate, $|\nabla p|$ and $|\nabla \rho|$ (if the flamelet structure is resolved). Readers interested in Lewis number effects in premixed turbulent combustion are referred to a review by Lipatnikov \& Chomiak (2005).

\subsection{Scalar Gradients and Principal Strain Rate: An Alignment Issue}

The increase in the area of a flame-front surface by turbulent stretching is often considered via an analogy with the exponential growth of the area of a material surface in homogeneous turbulence (Batchelor 1952). The latter effect results from statistical predominance of positive surface strain rate, $a_{t}$, and is closely related to the preferential alignment of scalar gradients with the most compressive principal strain rate (PSR) in homogeneous turbulence (e.g., Ashurst et al. 1987). Accordingly, the alignment of the local normal $\mathbf{n}$ to a flame front and eigenvectors of $S_{i j}$ is of paramount importance for fundamental understanding of the flame-turbulence interaction.

Over a decade ago, Swaminathan \& Grout (2006) analyzed DNS data obtained earlier from an SUF associated with the flamelet combustion regime. They documented the preferential alignment of $\mathbf{n}$ with the most extensive (largest positive) PSR within flamelets due to the local dilatation and hypothesized that turbulence could reduce scalar gradients in the flamelet combustion regime, contrary to an increase in scalar gradients by turbulent stretching in constant-density flows. This hypothesis triggered subsequent research into the alignment issue.

Chakraborty \& Swaminathan (2007) analyzed the same data and DNS data obtained from another SUF that propagated in intense $\left(u^{\prime} / S_{\mathrm{L}}=7.6\right)$ small-scale turbulence and was not associated with the flamelet regime. They concluded that the aforementioned unusual alignment and mitigation of scalar gradients by turbulence were specific to the flamelet regime, whereas $\mathbf{n}$ was aligned with the most compressive PSR in the latter flame. DNS data by Hamlington et al. (2011) support this conclusion.

Hartung et al. (2008, p. 1) experimentally confirmed the preferential alignment of $\mathbf{n}$ with the most extensive PSR within flamelets in five open lean ethylene-air flames stabilized on a bluff body and concluded that this "result questions the validity of passive scalar turbulence physics commonly used for premixed flame modeling."

Chakraborty et al. (2009) studied DNS data obtained from four SUFs characterized by various $L e$ and reported the preferential alignment of $\mathbf{n}$ with the most extensive PSR within flamelets, with the effect increased with decreasing $L e$. However, for isosurfaces $c(\mathbf{x}, t)=0.1$ associated with the flamelet leading edge and weak dilatation, $\mathbf{n}$ aligned preferentially with the most compressive PSR, similar to constant-density turbulence.

The latter result is important because flamelets propagate into unburned gas; hence, the flamelet-surface area and burning rate are increased by turbulence in the unburned gas. Accordingly, Steinberg et al. (2012) experimentally investigated three flames associated with the flamelet combustion regime and studied the alignment of $\mathbf{n}$ with eigenvectors of $S_{i j}$ at a flame contour precisely ahead of a zone of substantial dilatation. Similarly to mixing in constant-density turbulence, the most extensive PSR "was found to preferentially align perpendicular to the scalar gradients" (Steinberg et al. 2012, p. 2586), and turbulence was concluded to increase $|\nabla c|$.
Surface strain rate $\left(a_{t}\right)$ : projection of the rate-of-strain tensor $S_{i j}$ on the plane tangential to the surface

Principal strain rate (PSR): eigenvector of the rate-of-strain tensor 
Sponfeldner et al. (2015) experimentally investigated a single flame associated with the flamelet combustion regime and measured the angle between $\mathbf{n}$ and the most extensive PSR at different distances ahead of a flame contour characterized by the maximum gradient of the $\mathrm{OH}$ planar laser-induced fluorescence signal. Results show the lack of alignment far from the flame contour, the preferentially perpendicular alignment of $\mathbf{n}$ with respect to the most extensive PSR at distances from three to four laminar flame thicknesses, where dilatation vanishes, but the preferential alignment of $\mathbf{n}$ with the most extensive PSR in the vicinity of the flame contour, where dilatation is substantial.

Thus, as far as the interaction of scalar gradients in premixed flames with upstream turbulence in an unburned mixture is concerned, recent experimental data (Steinberg et al. 2012, Sponfeldner et al. 2015) have not revealed substantial qualitative peculiarities when compared to mixing in constant-density turbulence. Therefore, results discussed in this section do not put the concept of an increase in the area of a flame front by turbulent stretching into question. The significant influence of dilatation on the alignment of $\mathbf{n}$ and PSR vectors within flamelets is important for turbulent combustion models that deal with a transport equation for the mean scalar dissipation rate (Swaminathan \& Bray 2011).

Recently, Dopazo et al. (2015) analyzed DNS data obtained from a single SUF associated with the flamelet combustion regime and pointed out that the dilatation-induced dissipation of $|\nabla c|$ within a flamelet, highlighted by Swaminathan \& Grout (2006), was locally counteracted by a negative combustion-induced normal strain rate (i.e., an increase in self-propagation speeds of isosurfaces from cold to hot edges of the flamelet).

\subsection{Unburned Mixture Flow Upstream a Flame Front}

Because a flame front propagates into unburned gas, perturbations of the velocity field ahead of the front are required for the thermal expansion of the flow to affect the flame speed. Such an influence of thermal expansion on some components of the tensor $\left(\overline{u_{i}^{\prime} u_{j}^{\prime}}\right)_{\mathrm{u}}$ was documented in a few earlier experimental studies. In particular, Videto \& Santavicca (1990) reported a gradual increase in $u^{\prime}$ measured ahead of a mean flame brush as the flame approached the measurement point, with the local velocity spectrum changed at large wave numbers. Furukawa et al. (2002) documented larger $u_{\mathrm{u}}^{\prime}$ in flames than in the counterpart cold flows.

The influence of thermal expansion on the turbulent flow of unburned gas is often reduced to the DL instability of flame fronts. In the laminar case, the instability was predicted by Darrieus (1938) and Landau (1944) by analyzing the response of a planar flame front to infinitesimal 2D perturbations. The instability is caused by pressure perturbations induced in the unburned gas due to thermal expansion at a wrinkled flame front, which make the flow converged (diverged) upstream of concave (convex) flame-front bulges, thus increasing (decreasing) $u$ with respect to $S_{\mathrm{L}}$ and promoting further growth of the bulges.

The contribution of the local DL instability of flame fronts in turbulent flows to $S_{t}$ was hypothesized a long time ago (e.g., Scurlock \& Grover 1953). Both analytical models (Kuznetsov \& Sabelnikov 1990, Bychkov 2003) and a closure relation (Paul \& Bray 1996) for RANS simulations were developed to allow for this effect. Moreover, certain experimental data (e.g., Kobayashi et al. 1996) are often claimed to show an important role played by the DL instability in premixed turbulent burning. Nevertheless, this role is still a controversial issue (e.g., Klimenko 1998, Lipatnikov \& Chomiak 2005, 2010).

Although the DL instability appears to affect weakly turbulent $\left(u^{\prime} \approx S_{\mathrm{L}}\right)$ premixed flames, such effects are impeded by an increase in $u^{\prime} / S_{\mathrm{L}}$. For instance, Lipatnikov \& Chomiak (2005) pointed out that turbulent strains mitigated the instability by flattening local flame-front wrinkles. They 
compared $a_{t}$ with the instability growth rate and argued that the DL instability could play a substantial role in turbulent combustion only if the Karlovitz number $K a=\left(\delta_{L} / \eta\right)^{2}$ were less than a critical value, $K a_{\mathrm{cr}}$, with $K a_{\mathrm{cr}}=K a_{\mathrm{cr}}(\sigma)$ being significantly smaller than unity. Chaudhuri et al. (2011) obtained a similar criterion by comparing the local growth rates of a flame-front surface due to the instability and turbulence. Here, $\eta=\overline{\left(v_{\mathrm{u}}^{3} / \bar{\varepsilon}\right)^{1 / 4}}$ is the Kolmogorov length scale, i.e., the scale of the smallest eddies in a turbulent flow, and $\bar{\varepsilon}=2 v_{\mathrm{u}} \overline{S^{2}}$ is the rate of dissipation of TKE.

Recently, Creta \& Matalon (2011) and Fogla et al. (2015) performed 2D simulations of flamefront propagation in fluctuating velocity fields and identified two combustion modes using the following method. If the linear dependence of the flame-front speed $\mathcal{S}$ on the local stretch rate $\dot{s}=\Sigma^{-1} \mathrm{~d} \Sigma / \mathrm{d} t$ (with $\Sigma$ an infinitesimal element of the flame-front surface) is introduced based on the theory of weakly perturbed laminar premixed flames (e.g., Matalon 2007), then the front is stable with respect to small-scale perturbations whose wavelength $\lambda$ is smaller than a neutral wavelength $\lambda_{c}$, which is controlled by the Markstein length $L_{M}=(\mathrm{d} \mathcal{S} / \mathrm{d} \dot{s})_{s \rightarrow 0}$. Therefore, by varying $L_{\mathrm{M}}$, one can study supercritical and subcritical combustion modes. In the former mode, $\lambda_{c}$ is sufficiently small when compared to the width of the computational domain, and the DL instability can manifest itself in the simulations. In the latter case, $\lambda_{c}$ is large and computed results are not affected by the instability. Comparison of results obtained in such modes (two different $L_{\mathrm{M}}$, with all other things equal) shows that the local DL instability plays a substantial (minor) role in weakly (moderately) turbulent combustion associated with $u^{\prime} / S_{\mathrm{L}}<1\left(u^{\prime} / S_{\mathrm{L}}>1\right)$ (Fogla et al. 2015, figures 2 and 3).

Troiani et al. (2015) provided further support for Fogla et al.'s (2015) results by measuring $S_{t}$ in $\mathrm{C}_{3} \mathrm{H}_{8}$-air flames characterized by various $\Phi$ and $u^{\prime} / S_{\mathrm{L}}$. Experimental data reported in the form of $S_{t} / S_{\mathrm{L}}$ versus $u^{\prime} / S_{\mathrm{L}}$ showed two distinct branches, which were associated with the supercritical and subcritical modes. The two branches are observed even at $u^{\prime} / S_{\mathrm{L}}=4$. However, inspection of the data reveals that higher (lower) $S_{t}$ were obtained from rich (lean) flames characterized by faster molecular diffusion of the deficient (excess) reactant. Because differences in molecular diffusivities $D$ of reactants are well known to strongly affect $S_{t}$ (Lipatnikov \& Chomiak 2005), the two branches documented by Troiani et al. (2015) could be attributed not only to the DL instability, but also to the difference in $D$, at least in part.

Steinberg et al. (2009) simultaneously recorded (a) 2D images of the component $\omega_{z}$ of the vorticity vector normal to the image plane and $(b)$ the flame contour on this plane, with a time interval between two subsequent images equal to $0.9 \mathrm{~ms}$. By analyzing events associated with the wrinkling of an initially straight flame contour by a counter-rotating vortex pair, Steinberg et al. (2009) found that an initially negative correlation between locally positive $a_{t}$ and locally negative curvature $\kappa=\nabla \cdot \mathbf{n}$, which is typical for the interaction of a counter-rotating vortex pair with a laminar premixed flame, is commonly followed by a positive correlation between locally negative $a_{t}$ and $\kappa$. Because the DL solution predicts that $a_{t}<0$ and $\kappa<0$ ahead of an unstable laminar premixed flame, Steinberg et al. (2009, p. 1713) concluded that "the hydrodynamic instability mechanism caused significant strain on a flame and should be included in turbulent combustion models."

It is worth noting that the instability itself and the DL mechanism that causes it should be clearly distinguished. Indeed, the claim that the local DL instability plays an important role in premixed turbulent combustion implies at least indirectly that, for example, $U_{t}$ should depend on instability characteristics such as its growth rate or neutral wavelength. However, such a dependence has not yet been proven (Lipatnikov \& Chomiak 2005). Nevertheless, the DL mechanism does play a role in premixed turbulent combustion but manifests itself in phenomena that are not reduced to the evolution of an unstable flame in laminar flow. 

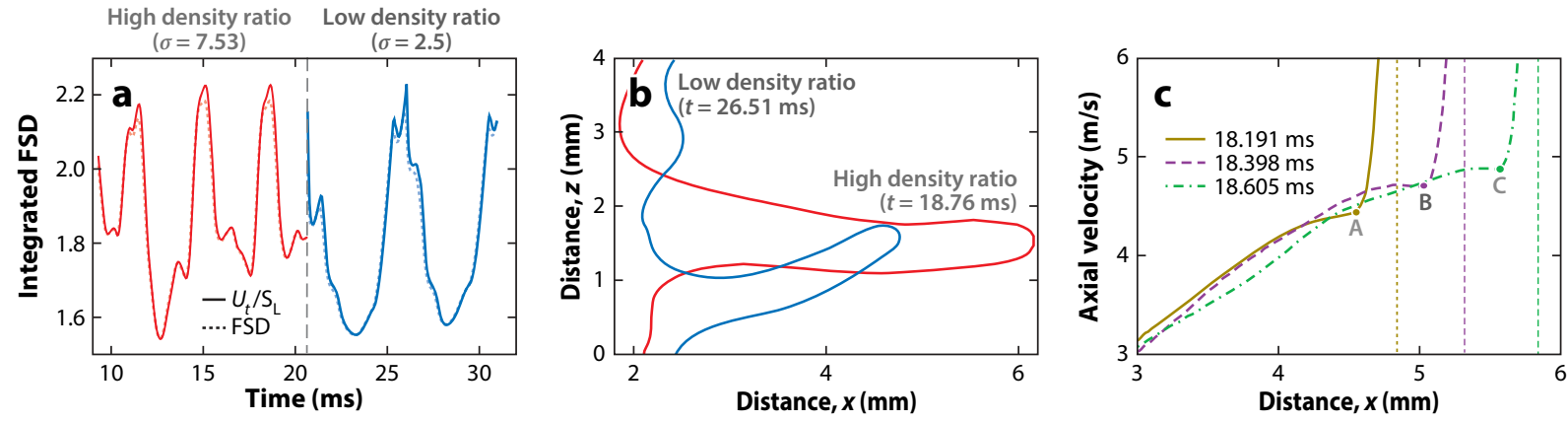

Figure 2

(a) Oscillations of $U_{t} / S_{\mathrm{L}}$ (solid lines) and integrated flame surface density (FSD) $\overline{|\nabla c|}$ (pale dotted lines) obtained with a high density ratio and low density ratio. (b) Isolines $c(x, z, t)=0.85$ in planes $y=$ const that cross the finger tip, computed with a high density ratio at $t=18.76 \mathrm{~ms}$ and a low density ratio at $t=26.51 \mathrm{~ms}$. Statistically planar 1D flames propagate from right to left. $(c)$ Growth of the axial flow velocity $u$ along the axis of an unburned mixture finger with a high density $(\sigma=7.53)$ ratio at various instants $t=t_{m}$. Vertical dashed lines show $x$ associated with the peak reaction rate along the finger axis at the instants $t_{m}$. Points $\mathrm{A}, \mathrm{B}$, and $\mathrm{C}$ indicate boundaries between unburned gas and flamelets.

For instance, DNS of SUFs (Bell et al. 2006; Poludnenko \& Oran 2010, 2011; Lipatnikov et al. 2015b; Poludnenko 2015) revealed large-scale oscillations of $U_{t}$ (see Figure $2 a$ ). A recent target-directed study of this phenomenon (Lipatnikov et al. 2015b) showed that peak values of $U_{t}(t)$ correlate with the appearance of an elongated unburned mixture finger (UMF) (see Figure $2 \boldsymbol{b}$ ) due to the high axial velocity of unburned gas near the finger tip (see points A, B, and $\mathrm{C}$ in Figure 2c). The UMF deeply intrudes into products, thus increasing $\delta_{t}$ and the flame surface area (see Figure 2a). Significant acceleration of the unburned gas within the finger is caused by the axial pressure gradient induced owing to thermal expansion at the finger sides. Finger-like structures have been documented in various experiments and DNS (e.g., see Figure 3).

Lipatnikov et al. (2015b) have argued that the UMF and the local DL instability of flame fronts in turbulent flow are different manifestations of the DL mechanism (i.e., the influence of pressure perturbations induced due to thermal expansion on the upstream flow of unburned gas), in particular, because the finger growth rate is an order of magnitude higher than the growth rates typical for the DL instability of laminar premixed flames. Recent DNS (Nivarti \& Cant 2015, figure 7) also supports the basic difference between the UMF and the local DL instability by showing fingers in very intense turbulence (e.g., $u^{\prime} / S_{\mathrm{L}}=30$ ), which is commonly associated with a negligible role played by the instability (Lipatnikov \& Chomiak 2005, Chaudhuri et al. 2011, Fogla et al. 2015). Furthermore, whereas the nonlinear stage of the DL instability has been studied in various $2 \mathrm{D}$ numerical simulations of laminar premixed flames with $L e=1$ (e.g., $\mathrm{Yu}$ et al. 2015a), we are not aware of observations of a UMF in such simulations. A laminar flame is stabilized due to cusp formation (Zel'dovich et al. 1985), but a cusp looks like the top of a triangle with a moderately acute angle (e.g., Yu et al. 2015a, figure 1) and differs significantly from a UMF.

The DL mechanism can manifest itself differently in laminar (the instability) and turbulent (UMF) premixed flames for the following reasons. First, pressure variations within a turbulent flame brush are significantly larger when compared to the counterpart laminar flame because the pressure drop across a fully developed SUF scales as $\tau \rho_{\mathrm{u}} S_{\mathrm{L}}^{2}$ and $\tau \rho_{\mathrm{u}} U_{t}^{2}$ in laminar and turbulent cases, respectively (Zel'dovich et al. 1985). Second, a UMF in a turbulent flow can be a manifestation of an instability with respect to finite perturbations, whereas laminar flames are unstable with respect to infinitesimal perturbations. 

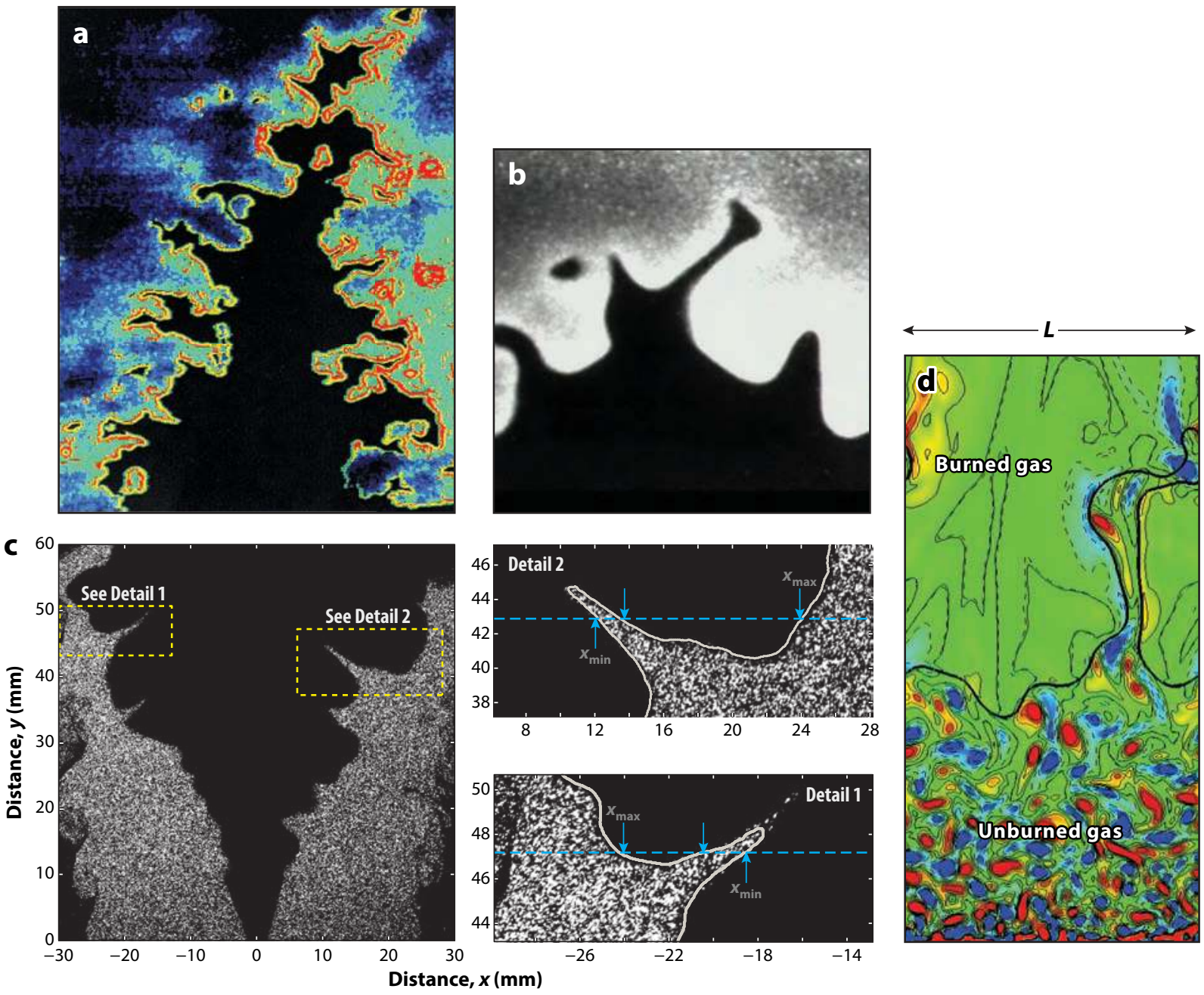

Figure 3

(a) $150 \times 100 \mathrm{~mm}^{2}$ and (b) $40 \times 40 \mathrm{~mm}^{2}$ OH planar laser-induced fluorescence images of a Bunsen flame and an open flame stabilized at a low-swirl burner. The unburned gas is dark in both panels. (c) Mie-scattering image of a V-shaped flame, in which the burned gas is dark. (d) 2D direct numerical simulation data obtained by Fogla et al. (2015) from a statistically planar flame. The solid black curve shows the flame front. Panel $a$ adapted from Buschmann et al. (1996) with permission from the Combustion Institute; panel $b$ adapted from Shepherd \& Cheng (2001) with permission from the Combustion Institute; panel $c$ adapted from Kheirkhah \& Gülder (2013), copyright 2013, AIP Publishing LLC; and panel $d$ adapted from Fogla et al. (2015) with permission from the Combustion Institute.

The above discussion implies that an increase in the density ratio should increase $U_{t}$ by increasing combustion-induced pressure variations within the flame brush and therefore the contribution of the DL mechanism to flame-surface-area production. Indeed, Figure $\boldsymbol{2} \boldsymbol{b}$ shows a longer UMF with a high density ratio when compared to that with a low density ratio. However, Figure $2 a$ indicates approximately the same flame surface areas and hence $U_{t} / S_{\mathrm{L}}$ in the two cases. The DL mechanism not only increases $U_{t}$ by creating a UMF, but also flattens the side surface of the UMF by inducing high-speed flow of unburned gas, which rapidly advects turbulent eddies to products, thus substantially reducing the residence time during which the eddies can wrinkle the 
finger surface (A.N. Lipatnikov, J. Chomiak, V.A. Sabelnikov, S. Nishiki \& T. Hasegawa, submitted manuscript). For instance, the side surface of the UMF with a high density ratio is planar in Figure $2 b$, whereas the UMF with a low density ratio is bent. It is unclear whether almost complete mutual cancellation of the two effects of the DL mechanism on $U_{t} / S_{\mathrm{L}}$, i.e., the same magnitudes of $U_{t} / S_{\mathrm{L}}$ computed at $\sigma=2.5$ and 7.53 , is occasional or it is controlled by a physical mechanism that should be revealed in future studies. Recent experiments with expanding statistically spherical premixed flames (A.N. Lipatnikov, W.Y. Li, L.J. Jiang \& S.S. Shy, submitted manuscript) did not show a difference in $S_{t}$ measured at $\sigma=4.95$ and 7.5 in a range of $u^{\prime} / S_{\mathrm{L}}=0.4-4.0$.

Combustion-induced pressure perturbations not only accelerate unburned gas, but also can affect the structure of the upstream turbulence by substantially changing the relative magnitudes of vorticity and strain-rate fluctuations. In constant-density turbulent flows, such relative magnitudes are often characterized using the $Q$ criterion (i.e., $Q=\omega^{2} / 4-S^{2} / 2$ ) (e.g., Tsinober 2009). $Q_{\mathrm{fu}}$ (i.e., values of $Q$ conditioned to an unburned flamelet edge) were recently analyzed by Lipatnikov et al. (2015a) by processing DNS data obtained from two flames characterized by different density ratios $\sigma$. Results show that $Q_{\mathrm{fu}}$ is always negative in the flame with the high density ratio $(\sigma=7.53)$, whereas the probability of $Q_{\mathrm{fu}}>0$ is substantial in the flame with the low density ratio $(\sigma=2.5)$ (see Figure $4 \boldsymbol{a}$ ). Moreover, Figure $4 \boldsymbol{b}$ shows that the total strain $S^{2}\left[\mathbf{x}_{\mathrm{f}}(t), t\right]$ conditioned to the leading edge of the instantaneous flame brush with the high density ratio is significantly larger than the total strain $\overline{S_{\mathrm{u}}^{2}}\left[x_{\mathrm{f}}(t), t\right]$ averaged over the leading plane $x=x_{\mathrm{f}}(t)$. Additionally, $\omega^{2}\left[\mathbf{x}_{\mathrm{f}}(t), t\right]$ is significantly smaller than $\overline{\omega_{\mathrm{u}}^{2}}\left[x_{\mathrm{f}}(t), t\right]$. Moreover, $Q\left[\mathbf{x}_{\mathrm{f}}(t), t\right]$ is highly negative, whereas $\bar{Q}_{\mathrm{u}}\left[x_{\mathrm{f}}(t), t\right]$ oscillates near zero (Lipatnikov et al. 2015a). With a low density ratio, differences in $Q\left[\mathbf{x}_{\mathrm{f}}(t), t\right]$ and $\bar{Q}_{\mathrm{u}}\left[x_{\mathrm{f}}(t), t\right]$ or in $S^{2}\left[\mathbf{x}_{\mathrm{f}}(t), t\right]$ and $\omega^{2}\left[\mathbf{x}_{\mathrm{f}}(t), t\right]$ are less pronounced, with both $S^{2}\left[\mathbf{x}_{\mathrm{f}}(t), t\right]$ and $\omega^{2}\left[\mathbf{x}_{\mathrm{f}}(t), t\right]$ smaller than $\overline{S_{\mathrm{u}}^{2}}\left[x_{\mathrm{f}}(t), t\right]$ and $\overline{\omega_{\mathrm{u}}^{2}}\left[x_{\mathrm{f}}(t), t\right]$, respectively. These recent results imply that, due to combustion-induced pressure perturbations, the velocity field seen by a premixed flame differs significantly from turbulence in the upstream flow far ahead of the flame brush.

Although the above discussion is limited to local manifestations of the DL mechanism, let us consider the global behavior of an unbounded SUF. Because, for any $\delta_{t}$, as large as we want, there
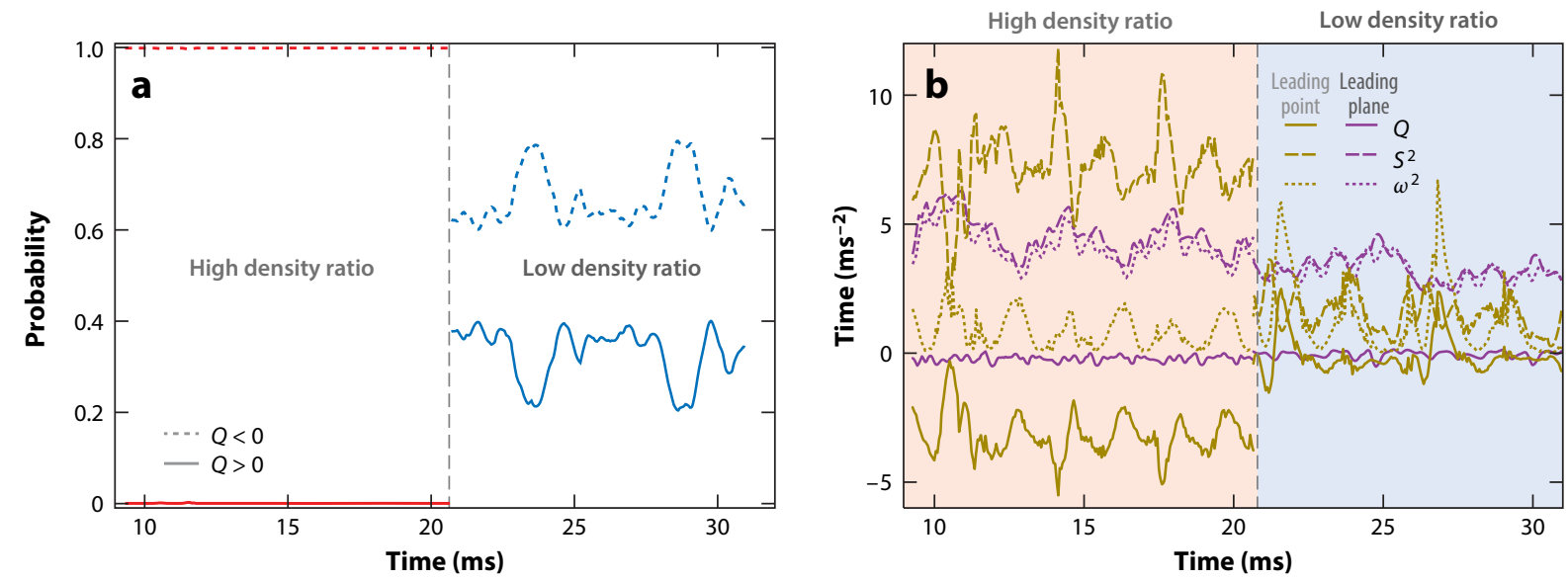

Figure 4

Results of direct numerical simulations of statistically planar 1D premixed turbulent flames. (a) Probabilities of $Q>0$ and $Q<0$ with a high density ratio and low density ratio, conditioned to unburned flamelet edges. (b) $Q$ criterion, total strain $S^{2}$, and enstrophy $\omega^{2}$ averaged in the vicinity of the leading points $\mathbf{x}_{\mathrm{f}}(t)$, with a high density ratio and low density ratio, and over leading planes $x=x_{\mathrm{f}}(t)$, with a high density ratio and low density ratio. 
is a wave number $\lambda_{1} \gg \delta_{t}$, the thick flame brush can be considered to be infinitely thin with respect to such a large-scale perturbation, and DL analysis can be applied to the entire turbulent flame by substituting $S_{\mathrm{L}}$ with $S_{t}$. Therefore, such an unbounded turbulent flame is hydrodynamically unstable with respect to the $\lambda_{1}$ perturbation (i.e., both $S_{t}$ and $\delta_{t}$ should grow with time). When $\delta_{t}$ becomes comparable with $\lambda_{1}$, the flame is stabilized with respect to the $\lambda_{1}$ perturbation due to nonlinear effects. However, the "stabilized" flame is unstable with respect to another perturbation with $\lambda_{2} \gg \delta_{t}=O\left(\lambda_{1}\right)$. Thus, both $S_{t}$ and $\delta_{t}$ should permanently grow if $\sigma>1$. Due to such a global DL instability of the entire flame brush, $S_{t}$ and $\delta_{t}$ obtained in DNS of SUFs should be sensitive to the width of the computational domain, but we are not aware of research into the issue.

There are other issues associated with the influence of the DL instability on DNS results. A typical DNS starts by embedding a planar 1D laminar flame into precomputed turbulence. Alternatively, one could simulate the laminar flame subject to the DL instability, obtain the curved flame surface stabilized due to nonlinear effects, and then embed this curved laminar flame into the turbulence. Under what conditions (if any) will the results of the two DNS started from different initial conditions be statistically identical after a transition period? Will the mean flame brush be statistically planar or curved, e.g., due to the aforementioned global DL instability? These issues have not yet been studied.

\section{TURBULENT TRANSPORT IN PREMIXED FLAMES}

\subsection{Phenomenology of Countergradient Transport}

The influence of thermal expansion on turbulent transport in flames manifests itself in countergradient transport (CGT), i.e., a positive scalar product $\overline{\rho \mathbf{u}^{\prime \prime} c^{\prime \prime}} \cdot \nabla \tilde{c}$ of the turbulent flux and mean scalar gradient vectors. In contrast, in constant-density nonreacting flows, the turbulent transport of a scalar fluid characteristic $q$ is commonly gradient and is modeled invoking the gradient diffusion closure (e.g., Hinze 1975), i.e., $\overline{\mathbf{u}^{\prime} q^{\prime}}=-D_{t} \nabla \bar{q}$ and $\overline{\mathbf{u}^{\prime} q^{\prime}} \cdot \nabla \bar{q}<0$, where $D_{t}>0$ is the turbulent diffusivity.

Although equations that predicted CGT in turbulent premixed flames were obtained by Prudnikov (1960) in the 1960s, target-directed research into this phenomenon was triggered by a theoretical study by Clavin \& Williams (1979), a model by Libby \& Bray (1981), and experimental evidence reported by Moss (1980) and Yanagi \& Mimura (1981). Subsequently, CGT was documented in various experimental and DNS investigations of premixed turbulent combustion, reviewed by Lipatnikov \& Chomiak (2010).

Equation 3 shows that $\left(\overline{\mathbf{u}}_{\mathrm{b}}-\overline{\mathbf{u}}_{\mathrm{u}}\right) \cdot \nabla \tilde{c}>0$ in the CGT case; that is, the magnitude $\left|\bar{u}_{n, \mathrm{~b}}\right|$ of the normal (to the flame brush) velocity $\overline{\mathbf{u}}_{\mathrm{b}} \cdot \nabla \bar{c} /|\nabla \bar{c}|$ conditioned to products should be larger than the counterpart quantity $\left|\bar{u}_{n, \mathrm{u}}\right|$ conditioned to unburned gas. Such a difference in the conditioned velocities is commonly attributed to two closely related physical mechanisms.

First, due to thermal expansion, the normal gas velocity increases from unburned to burned edges of a laminar premixed flame (Zel'dovich et al. 1985), and similar jumps in $|\mathbf{u} \cdot \mathbf{n}|$ occur locally at flame fronts in turbulent flows. That is, the pressure drop $\delta p_{\mathrm{f}}$ at the front results locally in $|\mathbf{u} \cdot \mathbf{n}|_{\mathrm{b}, \mathrm{f}}>|\mathbf{u} \cdot \mathbf{n}|_{\mathrm{u}, \mathrm{f}}$, thus contributing to $\left|\bar{u}_{n, \mathrm{~b}}\right|>\left|\bar{u}_{n, \mathrm{u}}\right|$.

Second, the mean pressure gradient $\nabla \bar{p}$ induced within the mean flame brush due to thermal expansion accelerates lighter products more than denser unburned gas (Scurlock \& Grover 1953, Libby \& Bray 1981) because $D \mathbf{u} / D t \propto \rho^{-1} \nabla p$ due to Navier-Stokes equations. This physical mechanism also contributes to $\left|\bar{u}_{n, \mathrm{~b}}\right|>\left|\bar{u}_{n, \mathrm{u}}\right|$.

In turbulent flow, the two aforementioned mechanisms associated with thermal expansion are counteracted by velocity fluctuations, which yield turbulent diffusion and therefore
Countergradient transport (CGT): turbulent transport of a mixture characteristic (temperature, concentration, etc.) in the direction of an increase in its mean value 
Gradient transport (GT): turbulent transport of a mixture characteristic in the direction of a decrease in its mean value $\bar{c}(1-\bar{c})\left(\overline{\mathbf{u}}_{\mathrm{b}}-\overline{\mathbf{u}}_{\mathrm{u}}\right) \cdot \nabla \bar{c}=\overline{\mathbf{u}^{\prime} c^{\prime}} \cdot \nabla \bar{c}=-D_{t} \nabla \bar{c} \cdot \nabla \bar{c}<0$ in the case of an interface that propagates in constant-density turbulent flow (Yu et al. 2014, 2015b). Accordingly, depending on the conditions, both CGT and gradient transport (GT) associated with $\overline{\rho \mathbf{u}^{\prime \prime} c^{\prime \prime}} \cdot \nabla \tilde{c}<0$ can occur in premixed turbulent flames.

The transition from GT to CGT was the focus of research on turbulent transport in premixed flames after the seminal work of Bray (1995), who obtained the first criterion of the transition by comparing the magnitude $\tau S_{\mathrm{L}}$ of the normal velocity jump at a laminar premixed flame with the magnitude of the difference in the conditioned velocities due to turbulent diffusion, which was assumed to scale as $u^{\prime}$. The criterion states that CGT occurs if $N_{\mathrm{B}}=\tau S_{\mathrm{L}} /\left(2 \psi u^{\prime}\right)>1$, where an increasing unity-order function $\psi=\psi\left(L / \delta_{\mathrm{L}}\right)$ of a ratio of an integral length scale $L$ of turbulence to $\delta_{\mathrm{L}}$ was introduced by Veynante et al. (1997) by analyzing their 2D DNS data. The number $N_{\mathrm{B}}$ is the Bray number.

The first experimental studies of the transition from CGT to GT in premixed turbulent flames supported the $N_{\mathrm{B}}$ criterion. In particular, by varying the equivalence ratio $\Phi$ and inlet value of $u^{\prime}$, Kalt et al. (1998) and Frank et al. (1999) found that the magnitude of the countergradient flux increased by a ratio of $\tau S_{\mathrm{L}} / u^{\prime}$ and the transition from GT to CGT occurred at $N_{\mathrm{B}} \approx 1$ if $\psi=0.5$. Similar trends were recently documented by Troiani et al. (2009), but they also reported an increase in $\psi$ by $\Phi$ in lean $(\Phi<1)$ mixtures. However, there are experimental and DNS data that do not support the $N_{\mathrm{B}}$ criterion. For instance, 2D DNS data by Veynante \& Poinsot (1997) indicated that the mean pressure gradient directed from products to unburned gas impeded CGT. Readers interested in more detailed discussion of the $N_{\mathrm{B}}$ criterion, its earlier tests, and modifications are referred to the review by Lipatnikov \& Chomiak (2010, section 5.1).

Three recent developments in the transition-criterion issue are worth noting. First, by comparing magnitudes of local velocity gradients induced by small-scale turbulent eddies and thermal expansion in the laminar premixed flame, Mura \& Champion (2009) argued that CGT occurs if $\tau S_{\mathrm{L}} / u^{\prime}>D a^{-1 / 2}$, where $D a=L S_{\mathrm{L}} /\left(u^{\prime} \delta_{\mathrm{L}}\right)$ is the Damköhler number. This result has been validated by considering four flames, with the $N_{\mathrm{B}}$ criterion also able to yield the correct direction of the flux $\overline{\rho \mathbf{u}^{\prime \prime} c^{\prime \prime}}$ in those flames.

Second, DNS data by Chakraborty \& Cant (2009) and Chakraborty \& Lipatnikov (2013) show that the flux $\overline{\rho \mathbf{u}^{\prime \prime} c^{\prime \prime}}, \psi$ in $N_{\mathrm{B}}, \overline{\mathbf{u}}_{\mathrm{u}}$, and $\overline{\mathbf{u}}_{\mathrm{b}}$ are substantially affected by the Lewis number (e.g., an increase in Le impedes CGT). Local variations in the mixture composition, enthalpy, and burning rate in flamelets stretched by turbulent eddies manifest themselves globally in a significant increase in turbulent burning velocity $U_{t}$ with decreasing $L e$, as reviewed by Lipatnikov \& Chomiak (2005). Therefore, a decrease in Le results in increasing $|\nabla \bar{p}|$ within the mean flame brush, thus promoting CGT. Moreover, a decrease in $L e$ results in increasing $\left|\delta p_{\mathrm{f}}\right|$ at stretched flamelets, which statistically dominate at the leading half $(\bar{c}<0.5)$ of the mean flame brush, thus also promoting CGT.

Third, the direction of the scalar flux is affected by premixed turbulent flame development (Lipatnikov \& Chomiak 2010). For instance, within the framework of the BML approach, Lipatnikov (2011c) integrated the well-known transport equation for $\overline{\rho c^{\prime \prime 2}}$ (Bray 1995) over an SUF. He assumed that $\int_{-\infty}^{\infty} \overline{\rho \mathbf{u}^{\prime \prime} c^{\prime \prime}} \cdot \nabla \bar{c} \mathrm{~d} x$ vanished at the CGT-GT transition because CGT and GT were characterized by opposite signs of the integrated product and arrived at an exact analytical integral criterion of the transition. The criterion compares $\int_{-\infty}^{\infty} \overline{2 c^{\prime \prime}\left[w_{c}+\nabla \cdot(\rho D \nabla c)\right]} \mathrm{d} x$ with the time derivative of $\int_{-\infty}^{\infty} \overline{\rho c^{\prime \prime 2}} \mathrm{~d} x$, thus clearly indicating an important role played by turbulent flame development. A simplified criterion developed by Lipatnikov (2011c) by closing the two integrals is consistent with certain experimental and DNS data; for example, it can yield the transition from CGT to GT with a decreasing equivalence ratio in lean mixtures, in line with experiments by Kalt 
et al. (1998), Frank et al. (1999), and Troiani et al. (2009). However, this model predicts neither the Lewis number nor the external pressure gradient effects.

Thus, even though (a) more physical mechanisms that affect the transition from CGT to GT have recently been studied and $(b)$ the exact, but unclosed, integral criterion has been derived, the CGT-GT transition issue still challenges the combustion community and requires further study.

\subsection{Modeling of Countergradient Transport in Premixed Flames}

Early models of CGT in premixed turbulent flames (e.g., Libby \& Bray 1981) dealt with a transport equation for the flux $\overline{\rho \mathbf{u}^{\prime \prime} c^{\prime \prime}}$ and invoked various assumptions to close it. This approach dominated the literature in the 1980s and 1990s, with the obtained results critically discussed recently (Lipatnikov \& Chomiak 2010, section 4.1). Briefly summarizing, various closed equations that involved a set of model constants were proposed, but none was validated against a wide set of substantially different experimental or DNS data by retaining all the constants unchanged. Moreover, if a closure relation obtained by a research group was subsequently tested by other experts by processing other DNS data, there were data that contradicted this closure relation at least quantitatively. Over the past decade, interest in this approach was reduced, but modeling of CGT in premixed turbulent flames developed in two alternative directions.

First, Im et al. (2004) considered the case of unburned and burned gases separated by an infinitely thin flame front and obtained unclosed transport equations for $\overline{\mathbf{u}}_{\mathrm{u}}$ and $\overline{\mathbf{u}}_{\mathrm{b}}$. A solution to these equations allows one to evaluate $\overline{\rho \mathbf{u}^{\prime \prime} c^{\prime \prime}}$ using Equation 3. Subsequently, Lee \& Huh (2004) and Lee et al. (2005) closed these equations and validated them in RANS simulations of two IJFs experimentally investigated by Cheng \& Shepherd (1991) and by Cho et al. (1988) and a single SUF studied in DNS by Nishiki et al. (2002, 2006).

Lipatnikov (2008) derived transport equations for $\overline{\mathbf{u}}_{\mathrm{u}}$ and $\overline{\mathbf{u}}_{\mathrm{b}}$ in a different form using the BML approach. Then Lipatnikov (2011b) closed various terms in these equations and validated the model jointly with Equation 3 against experimental data obtained from six IJFs (see Figures 5 and 6), including the two IJFs studied by Cheng \& Shepherd (1991) and Cho et al. (1988). Moreover, the model was applied to simulations of the transition from CGT to GT in SUFs (Lipatnikov 2011c). Results show that the transition is substantially affected by the flame development and is impeded by an increase in $S_{\mathrm{L}}$ or by a decrease in $u^{\prime}$, in line with experimental data by Kalt et al. (1998), Frank et al. (1999), and Troiani et al. (2009) and DNS data by Veynante et al. (1997).

It is worth stressing, however, that the aforementioned tests of the models that dealt with the transport equations for $\overline{\mathbf{u}}_{\mathrm{u}}$ and $\overline{\mathbf{u}}_{\mathrm{b}}$ were performed by numerically solving 1D RANS equations, whereas the approach has not yet been applied to simulations of statistically $2 \mathrm{D}$ or $3 \mathrm{D}$ turbulent flames. This obvious gap should be filled. Moreover, relations proposed to close each term in the considered equations should be investigated by processing DNS data computed under substantially different conditions. Thus, although first applications of the approach yielded encouraging results, there is a need for further tests.

Second, several models that yielded simple expressions for evaluating the flux $\overline{\rho \mathbf{u}^{\prime \prime} c^{\prime \prime}}$ were proposed over the past decade. Earlier, simple flux models were also developed. For example, Chomiak \& Nisbet (1995, p. 371) drew an analogy between the influence of thermal expansion on turbulent transport in premixed flames and the "motion of large-scale turbulence thermals." Veynante et al. (1997) highlighted the local normal velocity jump at the flame front. Bray et al. (2000) analyzed various terms in the transport equation for $\overline{\rho \mathbf{u}^{\prime \prime} c^{\prime \prime}}$ to obtain an algebraic expression for evaluating $\overline{\rho u^{\prime \prime} c^{\prime \prime}}$ in an IJF. Zimont \& Biagioli (2002) and Biagioli \& Zimont (2002) highlighted the preferential acceleration of the burned mixture by $\nabla \bar{p}$. All these models, with the exception of 

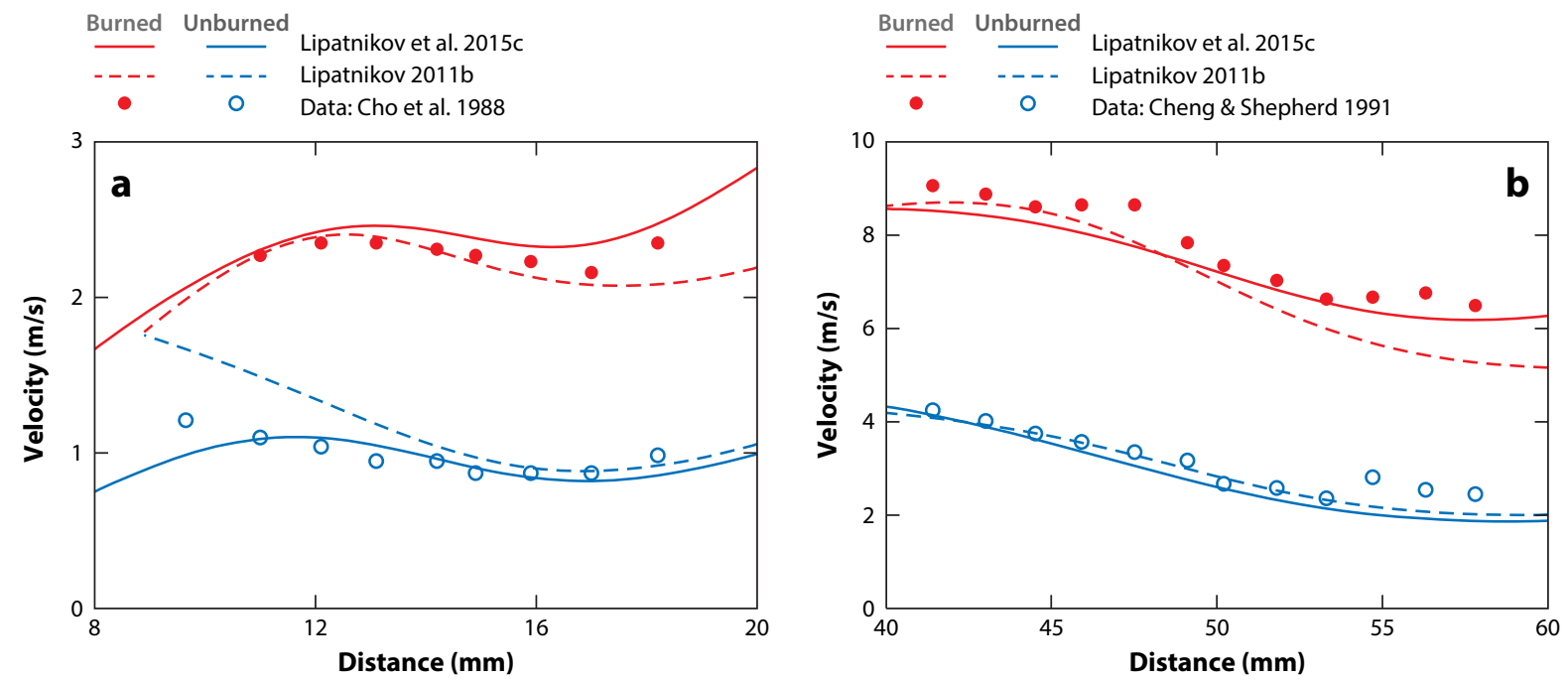

Figure 5

Velocities conditioned to unburned and burned gases. Circles represent experimental data obtained from impinging-jet flames (products on the left). Dashed lines represent results computed by Lipatnikov (2011b) by solving conditioned balance equations, and solid lines represent results computed by Lipatnikov et al. (2015c) using Equation 6.

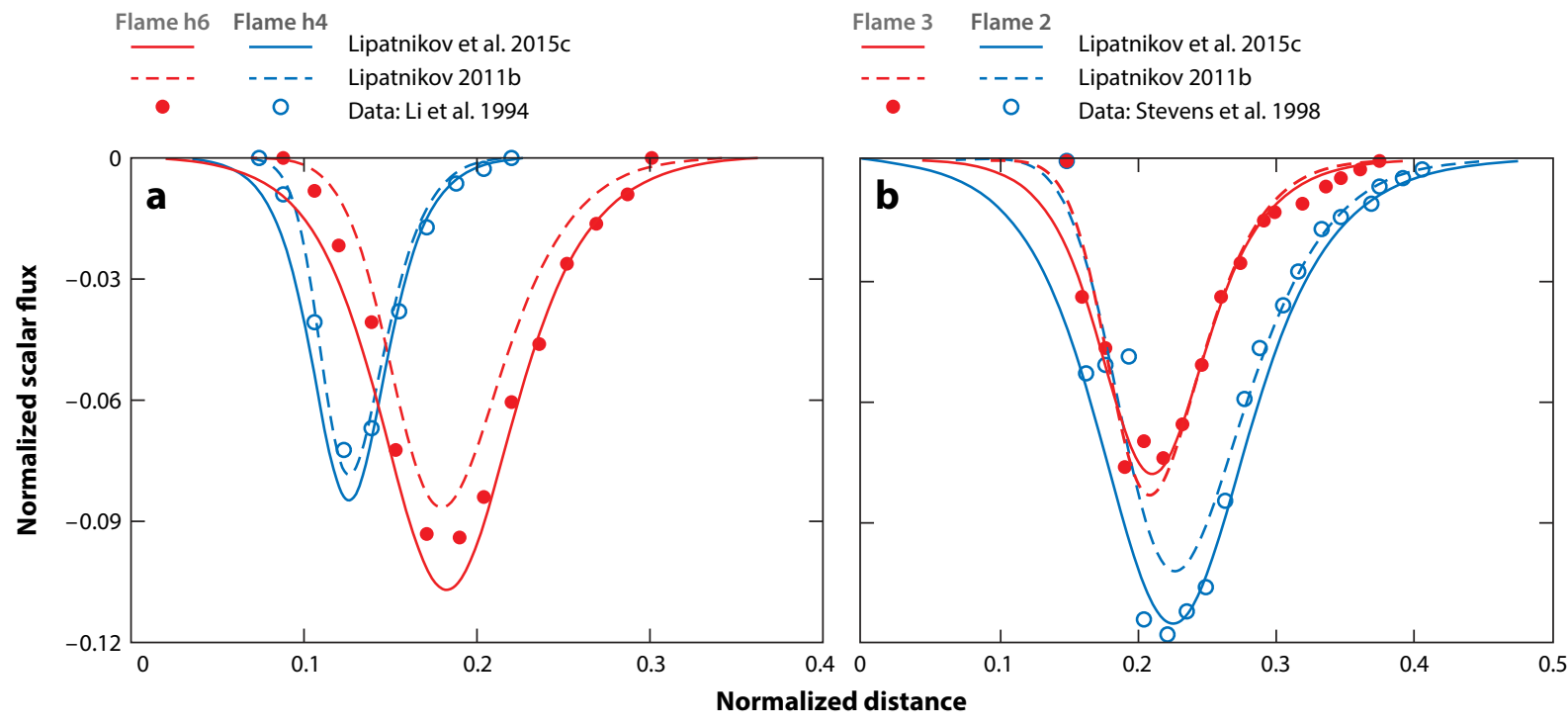

Figure 6

Turbulent scalar fluxes obtained (symbols) by (a) Li et al. (1994) and (b) Stevens et al. (1998) from impinging-jet flames (products on the left) and computed by Lipatnikov (2011b) by solving conditioned balance equations (dashed lines) and by Lipatnikov et al. (2015c) using Equation 6 (solid lines). Blue and red symbols/lines show results obtained from $(a) \mathrm{Li}$ et al.'s flames h4 and h6, respectively, and (b) Stevens et al.'s flames 2 and 3, respectively. 
Bray et al.'s, considered the flux $\overline{\rho \mathbf{u}^{\prime \prime} c^{\prime \prime}}$ to be a sum of two terms associated with turbulent diffusion and combustion-induced CGT, invoked the standard gradient diffusion approximation to evaluate the former term, and focused the analysis on closing the latter term. These four early models were critically discussed by Lipatnikov \& Chomiak (2010) and Sabelnikov \& Lipatnikov (2011).

Recently, Lecocq et al. (2010) extended the model by Veynante et al. (1997) and obtained an expression for evaluating subfiltered turbulent scalar flux in 3D LES of premixed burning. We are not aware of tests of this model against measured data on $\overline{\rho \mathbf{u}^{\prime \prime} c^{\prime \prime}}$ or $\overline{\mathbf{u}}_{\mathrm{u}}$.

Robin et al. (2010) decomposed the velocity field within a premixed turbulent flame brush into a turbulent contribution and direct thermal-expansion contribution due to the local normal velocity jump at the flame front. They assumed that the latter contribution scaled as $\tau S_{\mathrm{L}}$, invoked the gradient diffusion approximation to model the former contribution, and arrived at a simple algebraic closure relation for the flux $\overline{\rho \mathbf{u}^{\prime \prime} c^{\prime \prime}}$. Although the final result resembles earlier closure relations by Chomiak \& Nisbet (1995) and Veynante et al. (1997), there are some important differences. For instance, the model by Robin et al. (2010) includes an orientation factor that makes the direct thermal-expansion contribution less than $\tau S_{\mathrm{L}}$, and the authors aimed at excluding the influence of the thermal expansion on $\tilde{k}$ and its dissipation rate when evaluating the turbulent diffusivity.

Subsequently, Robin et al. $(2011,2012)$ extended the model. In particular, when evaluating the turbulent contribution, they allowed for preferential product acceleration by $\nabla \bar{p}$. The model was validated by analyzing DNS data obtained by Nishiki et al. $(2002,2006)$ from three SUFs characterized by significantly different density ratios, in 3D RANS simulations (Dong et al. 2013) of a single IJF experimentally studied by Cho et al. (1988), and in 2D RANS simulations of a confined conical flame stabilized by backward facing steps. The flame addressed in the 2D RANS simulations was experimentally studied by Besson et al. (2000), but the flux $\overline{\rho \mathbf{u}^{\prime \prime} c^{\prime \prime}}$ was not reported (i.e., that experimental validation of the model was indirect).

Sabelnikov \& Lipatnikov (2011, 2013a) considered the case of unburned and burned gases separated by an infinitely thin flame front and obtained the following simple equation:

$$
(1-\bar{c}) \nabla \cdot \overline{\mathbf{u}}_{\mathrm{u}}=\overline{\mathbf{u}_{\mathrm{u}} \cdot \mathbf{n}|\nabla c|}-\overline{\mathbf{u}}_{\mathrm{u}} \cdot \nabla \bar{c} \approx b \frac{(1-\bar{c})^{1 / 2}}{\left(1+u^{\prime} / S_{\mathrm{L}}\right)^{1 / 2}} \frac{u^{\prime}}{S_{\mathrm{L}}} \bar{W},
$$

which can be used jointly with Equation 3 to evaluate the flux $\overline{\rho \mathbf{u}^{\prime \prime} c^{\prime \prime}}$. The first equality in Equation 6 is exact in the considered case and results straightforwardly from the incompressibility of the unburned gas. The approximate equality is a closure relation obtained based on an analysis of DNS data by Im et al. (2004) and RANS simulations of SUFs characterized by various $u^{\prime} / S_{\mathrm{L}}$ (Sabelnikov \& Lipatnikov 2013a).

Subsequently, Equation 6 was tested against DNS data obtained from four SUFs characterized by different density ratios, ranging from $\sigma=2.5$ to 7.53 . The model well predicted the DNS data on $\bar{u}_{\mathrm{u}}(\bar{c})$ provided that the constant $b$ was substituted with the following linear fit: $b=$ $-0.18+0.35 \sigma$ (Lipatnikov et al. 2015c). The decrease in $b$ with decreasing $\sigma$ can be attributed to a decrease in the correlation $\overline{\mathbf{u}_{\mathrm{u}} \cdot \mathbf{n}|\nabla c|}$ because the effect of the flame front on the local flow is reduced when the density ratio is decreased.

In addition to the four $\bar{u}_{\mathrm{u}}(\bar{c})$ curves computed in DNS, the model supplemented with Equation 3 well predicts experimental data obtained from six IJFs (Lipatnikov et al. 2015c) (see Figures 5 and 6). Moreover, Lipatnikov \& Sabelnikov (2013) applied Equation 6 to RANS simulations of the transition from CGT to GT in developing SUFs and showed that CGT is impeded by an increase in $u^{\prime}$ and a decrease in $S_{\mathrm{L}}$, in line with experiments (Kalt et al. 1998, Frank et al. 1999, Troiani et al. 2009).

In the aforementioned papers, Equation 6 was tested by simulating flames described by statistically $1 \mathrm{D}$ transport equations. In such cases, a single scalar Equation 6 is sufficient to evaluate 
a single conditioned velocity $\bar{u}_{\mathrm{u}}$. This is not the case if two or three components of the vector $\overline{\mathbf{u}}_{\mathrm{u}}$ should be determined in a statistically $2 \mathrm{D}$ or $3 \mathrm{D}$ case. In recent 2D RANS simulations of open conical rim-stabilized (Bunsen) flames experimentally investigated by Frank et al. (1999) and Pfadler et al. (2008), Yasari \& Lipatnikov (2015) resolved the problem by invoking the gradient diffusion closure of the tangential (to the mean flame brush) component of the flux vector $\overline{\rho \mathbf{u}^{\prime \prime} c^{\prime \prime}}$ (i.e., the tangential flux vanished). In line with measurements by Pfadler et al. (2008), the model yielded CGT in the radial (almost normal to the mean flame brush) direction in all studied flames, with the magnitude of the flux substantially increased by the equivalence ratio $\Phi$ in lean $(\Phi<1)$ mixtures and weakly decreased with increasing inlet mass flow rate. In line with measurements by Frank et al. (1999), the model yielded a reduction of the magnitude of the CGT flux followed by a transition to GT at $\Phi=0.7$ when $\Phi$ was decreased from $\Phi=1$ to 0.6.

All in all, Equation 6 has been validated against a wide set of experimental and DNS data with encouraging test results. Therefore, the model deserves further study by applying it to other premixed turbulent flames.

\subsection{Effects of Countergradient Turbulent Transport on Flame Speed}

Although a reduction of the turbulent flame speed $S_{t}$ and mean flame brush thickness $\delta_{t}$ by CGT was discussed in various papers, there was a need for analytical expressions that predicted the dependencies of $S_{t}$ and $\delta_{t}$ on the CGT flux magnitude. Accordingly, Sabelnikov \& Lipatnikov (2013b, 2014, 2015) theoretically and numerically studied traveling wave (TW) solutions $\tilde{c}=$ $\tilde{c}\left(x+S_{t} t\right)$ to the generic $1 \mathrm{D}$ transport equation

$$
\left.\frac{\partial(\bar{\rho} \tilde{c})}{\partial t}+\frac{\partial(\bar{\rho} \tilde{u} \tilde{c})}{\partial x}=D_{t} \frac{\partial}{\partial x}\left(\bar{\rho} \frac{\partial \tilde{c}}{\partial x}\right)-V \frac{\partial[\bar{\rho} \tilde{c}(1-\tilde{c})]}{\partial x}+\frac{\rho_{\mathrm{u}} \tilde{c}(1-\tilde{c})}{\tau_{\mathrm{f}}} 1+2 \alpha \bar{c}\left(1-\frac{\sigma-1}{2 \sigma} \bar{c}\right)\right]
$$

supplemented with the BML equations $\rho_{\mathrm{b}} \bar{c}=\bar{\rho} \tilde{c}=\rho_{\mathrm{u}} \tilde{c} /[1+(\sigma-1) \tilde{c}]$ (Bray \& Moss 1977). Three terms on the right-hand side are associated with turbulent diffusion, combustion-induced CGT, and the mean rate of product creation, respectively, with the turbulent diffusivity $D_{t}$, the CGT flux magnitude $V$, the timescale $\tau_{\mathrm{f}}$, and the coefficient $\alpha \geq-\sigma /(1+\sigma)$ as input parameters. Variations in $\alpha$ offer an opportunity to consider both concave functions $f(\tilde{c})$ and functions with an inflection point, in which the function changes its shape from convex to concave. Using results of the theory of convection-reaction-diffusion equations (e.g., Ebert \& van Saarlos 2000), Sabelnikov \& Lipatnikov (2015, table 1) analytically found that the normalized physically relevant TW speed $\Lambda=S_{t} / \sqrt{D_{t} / \tau_{\mathrm{f}}}$ and thickness $d_{t}=\delta_{t} / \sqrt{D_{t} \tau_{\mathrm{f}}}$ were functions of $\alpha$, the density ratio $\sigma$, and the normalized CGT flux magnitude $N=V / \sqrt{D_{t} / \tau_{\mathrm{f}}}$. A physically relevant TW solution is the solution to the unsteady problem, reached at $t \rightarrow \infty$ provided that the initial conditions are compact, i.e., $\tilde{c}\left(x<x_{1}, 0\right)=0, \tilde{c}\left(x>x_{2}, 0\right)=1$, and $x_{1}<x_{2}$. The following results are worth emphasizing.

First, if $0 \leq N \leq N_{\mathrm{cr}}$, with $N_{\mathrm{cr}}=N_{\mathrm{cr}}(\alpha)$ analytically found, then one obtains $\Lambda=2-N$. Second, if $N_{\text {cr }} \leq N$, then one obtains $\Lambda=2(1+\alpha) /\left(N+\sqrt{N^{2}+4 \alpha}\right)$ and $d_{t}=4 \Lambda /(1+\alpha)$. Third, the direction of the total flux (i.e., the sign of the sum of the first and second terms on the right-hand side of Equation 7) depends not only on $N$, but also on $\alpha$, thus indicating that the use of a single Bray number is not sufficient to predict the flux direction. Finally, at $N=N_{\mathrm{cr}}$, the transition from pulled $\left(N<N_{\text {cr }}\right)$ to pushed $\left(N>N_{\text {cr }}\right)$ TW solutions occurs. In the former and latter cases, the TW speed $\Lambda$ is controlled by the behavior of various terms in Equation 7 at the leading edge $\bar{c} \rightarrow 0$ and in the entire flame brush, respectively. This finding emphasizes the importance of CGT in premixed turbulent flames because variations in the CGT flux magnitude 
not only change the flame speed quantitatively, but also change the fundamental nature of flame propagation (pulled or pushed TW).

\section{SUMMARY POINTS}

1. Contemporary models of premixed turbulent burning focus on the influence of turbulence on combustion. However, because the turbulent flame speed is mainly controlled by turbulence characteristics and is of the order of the rms turbulent velocity under typical conditions, and because the feedback influence of the combustion-induced thermal expansion on the incoming flow of unburned gas can manifest itself in various important effects discussed above and can substantially change the local turbulence characteristics, a deep understanding of the thermal-expansion effects is strongly required to increase predictive capabilities of physical and numerical models used to develop future ultraclean, highly efficient, and fuel-flexible internal combustion engines.

2. Pressure perturbations caused by thermal expansion within a premixed flame front can substantially affect the flow field behind the front (e.g., turbulence generation in combustion-induced shear layers), within the front (e.g., anisotropic vorticity generation due to baroclinic torque and vorticity attenuation due to dilatation), and ahead of the front (e.g., perturbations of the upstream velocity field).

3. Due to the intermittency of unburned and burned mixtures, separated by a thin zone that density, temperature, and mass fraction variations are localized to, neither mean nor conditioned second moments of the velocity field properly characterize turbulence within a premixed turbulent flame brush. Quantities controlled by velocity gradients, such as the mean total strain $\overline{S^{2}}$, seem to be more appropriate characteristics of turbulence in premixed flames.

4. The Darrieus-Landau mechanism appears to play a substantial role in premixed turbulent combustion by controlling the growth of unburned mixture fingers and therefore causing oscillations of the flame surface area, turbulent burning velocity, $U_{t}$, and mean flame brush thickness $\delta_{t}$. Moreover, pressure perturbations induced in the unburned gas due to the Darrieus-Landau mechanism can change the nature of the flow upstream of flame fronts.

5. Although the alignment between a flamelet normal and the local principal strain rates is changed within the flamelet due to dilatation if the local turbulence is sufficiently weak, this phenomenon does not seem to be of primary importance when studying the effects of upstream turbulent eddies on flamelet propagation into the unburned gas.

6. Both countergradient and gradient transport can occur in premixed turbulent flames, with the former associated with a larger laminar flame speed, higher density ratio, lower rms turbulent velocity, and smaller Lewis number.

7. First applications of recently developed simple models of turbulent scalar flux in premixed flames have yielded encouraging results.

8. Countergradient transport reduces the turbulent flame speed and mean flame brush thickness. Moreover, countergradient transport can change the fundamental nature of flame propagation (i.e., the flame speed can be controlled by processes localized either to the entire flame brush or to its leading edge if countergradient transport is strong or weak, respectively). 


\section{FU'TURE ISSUES}

1. Models should be developed that can predict basic turbulence characteristics such as the mean total strain $\overline{S^{2}}$ and enstrophy $\overline{\omega^{2}}$ in Reynolds-averaged Navier-Stokes and largeeddy simulation research into premixed turbulent combustion.

2. Because the vast majority of contemporary models of the influence of turbulence on the premixed burning rate (e.g., Lipatnikov \& Chomiak 2002) consider the canonical rms velocity $u^{\prime}$ to be the primary turbulence characteristic that mainly controls the influence, such models should be revisited in order to consider the mean rate $\bar{W}$ in Equation 5 to be controlled by fundamentally appropriate characteristics of turbulence within a premixed flame brush.

3. Because combustion-induced pressure perturbations affect the turbulent flow of unburned gas upstream of flame fronts, there is a need for target-directed research into the structure (e.g., relative magnitudes of enstrophy and strain rate, relative magnitudes of rotational and irrotational components of the flow velocity vector) of the upstream turbulence seen by the flame fronts.

4. A physical mechanism (if any) of a feedback effect of turbulence generated behind flame fronts on turbulent flame speed $S_{t}$ is still unknown. Although correlations between $S_{t}$ and canonical second moments of the velocity field $\mathbf{u}(\mathbf{x}, t)$ evaluated within a premixed turbulent flame brush were reported (e.g., Poludnenko 2015, figure 14), it is unclear whether such a correlation indicates burning acceleration due to flame-generated turbulence or the correlation shows an effect of $S_{t}$ on the rms turbulent velocity $u^{\prime}$, because the mean pressure gradient $\nabla \bar{p}$ depends on $S_{t}$ and causes an increase in $u^{\prime}$.

5. The effect of the density ratio on $S_{t}$ requires target-directed investigation.

6. In DNS of premixed turbulent flames, the influence of the computational domain width on turbulent flame speed $S_{t}$ is worth studying to gain insight into eventual DarrieusLandau instability of the entire turbulent flame brush.

7. A criterion for determining the direction of turbulent scalar flux in premixed flames has to be further developed to allow for the influence of the external pressure gradient, turbulent flame development, and Lewis number.

8. A role played by the leading edge of a premixed turbulent flame brush in its propagation requires target-directed study.

\section{DISCLOSURE STATEMENT}

The authors are not aware of any biases that might be perceived as affecting the objectivity of this review.

\section{ACKNOWLEDGMENTS}

V.A.S. gratefully acknowledges financial support provided by ONERA. A.N.L. gratefully acknowledges fruitful discussions with Prof. Jerzy Chomiak and financial support provided by the Chalmers Combustion Engine Research Center (CERC), Swedish Energy Agency, and Chalmers Energy and Transport Areas of Advance. 


\section{LITERATURE CITED}

Ashurst WT, Kerstein AR, Kerr RM, Gibson CH. 1987. Alignment of vorticity and scalar gradient with strain rate in simulated Navier-Stokes turbulence. Phys. Fluids 30:2343-53

Batchelor GK. 1952. The effect of homogeneous turbulence on material lines and surfaces. Proc. R. Soc. Lond. A 213:349-66

Bell JB, Day MS, Grcar JF, Lijewski MJ. 2006. Active control for statistically stationary turbulent premixed flame simulations. Commun. Appl. Math. Comput. Sci. 1:29-51

Besson M, Bruel P, Champion JL, Deshaies B. 2000. Experimental analysis of combusting flows developing over a plane-symmetric expansion. 7. Thermophys. Heat Transfer 14:59-67

Biagioli F, Zimont VL. 2002. Gasdynamics modelling of counter-gradient transport in open and impinging turbulent premixed flames. Proc. Combust. Inst. 29:2087-95

Bobbitt B, Lapointe S, Blanquart G. 2016. Vorticity transformation in high Karlovitz number premixed flames. Phys. Fluids 28:015101

Borghi R. 1988. Turbulent combustion modeling. Prog. Energy Combust. Sci. 14:245-92

Bray KNC. 1995. Turbulent transport in flames. Proc. R. Soc. Lond. A 451:231-56

Bray KNC, Champion M, Libby PA. 2000. Premixed flames in stagnating turbulence. Part IV: a new theory for the Reynolds stresses and Reynolds fluxes applied to impinging flows. Combust. Flame 120:1-18

Bray KNC, Libby PA. 1976. Interaction effects in turbulent premixed flames. Phys Fluids 19:1687-701

Bray KNC, Moss JB. 1977. A unified statistical model for the premixed turbulent flame. Acta Astronaut. 4:291-319

Buckmaster JD. 1993. The structure and stability of laminar flames. Annu. Rev. Fluid Mech. 25:21-53

Buschmann A, Dinkelacker F, Schäfer T, Schäfer M, Wolfrum J. 1996. Measurement of the instantaneous detailed flame structure in turbulent premixed combustion. Proc. Combust. Inst. 26:437-45

Bychkov V. 2003. Importance of the Darrieus-Landau instability for strongly corrugated turbulent flames. Phys. Rev. E 68:066304

Chakraborty N. 2014. Statistics of vorticity alignment with local strain rates in turbulent premixed flames. Eur. 7. Mech. B 46:201-20

Chakraborty N, Cant RS. 2009. Effects of Lewis number on scalar transport in turbulent premixed flames. Phys. Fluids 21:035110

Chakraborty N, Katragadda M, Cant RS. 2011. Effects of Lewis number on turbulent kinetic energy transport in premixed flames. Phys. Fluids 23:075109

Chakraborty N, Klein M, Swaminathan N. 2009. Effects of Lewis number on the reactive scalar gradient alignment with local strain rate in turbulent premixed flames. Proc. Combust. Inst. 32:1409-17

Chakraborty N, Konstantinou I, Lipatnikov AN. 2016. Effects of Lewis number on vorticity and enstrophy transport in turbulent premixed flames. Phys. Fluids 28:015109

Chakraborty N, Lipatnikov AN. 2013. Effects of Lewis number on conditional fluid velocity statistics in low Damköhler number turbulent premixed combustion: a direct numerical simulation analysis. Phys. Fluids 25:045101

Chakraborty N, Swaminathan N. 2007. Influence of the Damköhler number on turbulence-scalar interaction in premixed flames. I. Physical insight. Phys. Fluids 19:045103

Chaudhuri S, Akkerman V, Law CK. 2011. Spectral formulation of turbulent flame speed with consideration of hydrodynamic instability. Phys. Rev. E 84:026322

Chen JH, Lumley JL, Gouldin FC. 1986. Modeling of wrinkled laminar flames with intermittency and conditional statistics. Proc. Combust. Inst. 21:1483-91

Cheng RK, Shepherd IG. 1986. Interpretation of conditional statistics in open oblique premixed turbulent flames. Combust. Sci. Technol. 49:17-40

Cheng RK, Shepherd IG. 1991. The influence of burner geometry on premixed turbulent flame propagation. Combust. Flame 85:7-26

Cho P, Law CK, Cheng RK, Shepherd IG. 1988. Velocity and scalar fields of turbulent premixed flames in stagnation flow. Proc. Combust. Inst. 22:739-45

Chomiak J. 1990. Combustion: A Study in Theory, Fact and Application. New York: Gordon \& Breach 
Chomiak J, Nisbet JR. 1995. Modeling variable density effects in turbulent flames—some basic considerations. Combust. Flame 102:371-86

Clavin P. 1994. Premixed combustion and gas dynamics. Annu. Rev. Fluid Mech. 26:321-52

Clavin P, Williams FA. 1979. Theory of premixed-flame propagation in large-scale turbulence. F. Fluid Mech. 90:589-604

Creta F, Matalon M. 2011. Propagation of wrinkled turbulent flames in the context of hydrodynamic theory. 7. Fluid Mech. 680:225-64

Damköhler G. 1940. Der Einfuss der Turbulenz auf die Flammengeschwindigkeit in Gasgemischen. Z. Electrochem. 46:601-52

Darrieus G. 1938. Propagation d'un front de flamme. Presented at Tech. Mod., Paris

Dong HQ, Robin V, Mura A, Champion M. 2013. Analysis of algebraic closures of the mean scalar dissipation rate of the progress variable applied to stagnating turbulent flames. Flow Turbul. Combust. 90:301-23

Dopazo C, Cifuentes L, Martin J, Jimenez C. 2015. Strain rates normal to approaching iso-scalar surfaces in a turbulent premixed flame. Combust. Flame 162:1729-36

Driscoll JF. 2008. Turbulent premixed combustion: flamelet structure and its effect on turbulent burning velocities. Prog. Energy Combust. Sci. 34:91-134

Ebert U, van Saarlos W. 2000. Front propagation into unstable states: universal algebraic convergence towards uniformly translating pulled fronts. Physica D 146:1-99

Echekki T, Mastorakos E, eds. 2011. Turbulent Combustion Modeling. Berlin: Springer

Fogla N, Creta F, Matalon M. 2015. Effect of folds and pockets on the topology and propagation of premixed turbulent flames. Combust. Flame 162:2758-77

Frank JH, Kalt PAM, Bilger RW. 1999. Measurements of conditional velocities in turbulent premixed flames by simultaneous OH PLIF and PIV. Combust. Flame 116:220-32

Furukawa J, Noguchi Y, Hirano T, Williams FA. 2002. Anisotropic enhancement of turbulence in large-scale, low-intensity turbulent premixed propane-air flames. F. Fluid Mech. 462:209-43

Günther R. 1983. Turbulence properties of flames and their measurement. Prog. Energy Combust. Sci. 9:105-54

Hamlington PE, Poludnenko AY, Oran ES. 2011. Interactions between turbulence and flames in premixed reacting flows. Phys. Fluids 23:125111

Hartung G, Hult J, Kaminski CF, Rogerson JW, Swaminathan N. 2008. Effect of heat release on turbulence and scalar-turbulence interaction in premixed combustion. Phys. Fluids 20:035110

Hinze JO. 1975. Turbulence. New York: McGraw Hill. 2nd ed.

Im YH, Huh KY, Nishiki S, Hasegawa T. 2004. Zone conditional assessment of flame-generated turbulence with DNS database of a turbulent premixed flame. Combust. Flame 137:478-88

Kalt PAM, FrankJH, Bilger RW. 1998. Laser imaging of conditional velocities in premixed propane-air flames by simultaneous OH PLIF and PIV. Proc. Combust. Inst. 27:751-58

Karlovitz B, Denniston DW, Wells FE. 1951. Investigation of turbulent flames. F. Chem. Phys. 19:541-47

Kataoka I. 1986. Local instant formulation of two-phase flow. Int. F. Multiphase Flow 12:745-58

Kheirkhah S, Gülder ÖL. 2013. Turbulent premixed combustion in V-shaped flames: characteristics of flame front. Phys. Fluids 25:055107

Klimenko AY. 1998. Examining the cascade hypothesis for turbulent premixed combustion. Combust. Sci. Technol. 139:15-40

Kobayashi H, Tamura T, Maruta K, Niioka T, Williams FA. 1996. Burning velocity of turbulent premixed flames in a high-pressure environment. Proc. Combust. Inst. 26:389-96

Kolla H, Hawkes ER, Kerstein AR, Swaminathan N, Chen JH. 2014. On velocity and reactive scalar spectra in turbulent premixed flames. F. Fluid Mech. 754:456-87

Kuznetsov VR. 1979. Estimate of the correlation between pressure pulsations and the divergence of the velocity in subsonic flows of variable density. Fluid Dyn. 14:328-34

Kuznetsov VR, Sabelnikov VA. 1990. Turbulence and Combustion. New York: Hemisphere

Landau LD. 1944. On the theory of slow combustion. Acta Psysicochim. USSR 19:77-85

Lecocq G, Richard S, Colin O, Vervisch L. 2010. Gradient and counter-gradient modeling in premixed flames: theoretical study and application to the LES of a lean premixed turbulent swirl burner. Combust. Sci. Technol. 182:465-79 
Lee E, Huh KY. 2004. Zone conditional modeling of premixed turbulent flames at a high Damköhler number. Combust. Flame 138:211-24

Lee E, Im YH, Huh KY. 2005. Zone conditional analysis of a freely propagating one-dimensional turbulent premixed flame. Proc. Combust. Inst. 30:851-57

Li SC, Libby PA, Williams FA. 1994. Experimental investigation of a premixed flame in an impinging turbulent stream. Proc. Combust. Inst. 25:1207-14

Libby PA. 1975. On the prediction of intermittent turbulent flows. F. Fluid Mech. 68:273-95

Libby PA, Bray KNC. 1977. Variable density effects in premixed turbulent flames. AIAA F. 15:1186-93

Libby PA, Bray KNC. 1981. Countergradient diffusion in premixed turbulent flames. AIAA 7. 19:205-13

Librovich VB, Lisitzyn VI. 1977. Interaction of flow pulsations and chemical reaction in turbulent flames. AIAA 7. 15:227-33

Lipatnikov AN. 2008. Conditionally averaged balance equations for modeling premixed turbulent combustion in flamelet regime. Combust. Flame 152:529-47

Lipatnikov AN. 2009. Can we characterize turbulence in premixed flames? Combust. Flame 156:1242-47

Lipatnikov AN. 2011a. Conditioned moments in premixed turbulent reacting flows. Proc. Combust. Inst. 33:1489-96

Lipatnikov AN. 2011b. A test of conditioned balance equation approach. Proc. Combust. Inst. 33:1497-504

Lipatnikov AN. 2011c. Transient behavior of turbulent scalar transport in premixed flames. Flow Turbul. Combust. 86:609-37

Lipatnikov AN. 2012. Fundamentals of Premixed Turbulent Combustion. Boca Raton, FL: CRC

Lipatnikov AN, Chomiak J. 2002. Turbulent flame speed and thickness: phenomenology, evaluation, and application in multi-dimensional simulations. Prog. Energy Combust. Sci. 28:1-74

Lipatnikov AN, Chomiak J. 2005. Molecular transport effects on turbulent flame propagation and structure. Prog. Energy Combust. Sci. 31:1-73

Lipatnikov AN, Chomiak J. 2010. Effects of premixed flames on turbulence and turbulent scalar transport. Prog. Energy Combust. Sci. 36:1-102

Lipatnikov AN, Chomiak J, Sabelnikov VA, Nishiki S, Hasegawa T. 2015a. Influence of heat release in a premixed flame on weakly turbulent flow of unburned gas: a DNS study. Proc. 25th Int. Colloq. Dyn. Explos. Reactive Syst., ed. MI Radulescu, Pap. 74. http://www.engineering.leeds.ac.uk/short-courses/ ICDERS/documents/ICDERS074.pdf

Lipatnikov AN, Chomiak J, Sabelnikov VA, Nishiki S, Hasegawa T. 2015b. Unburned mixture fingers in premixed turbulent flames. Proc. Combust. Inst. 35:1401-8

Lipatnikov AN, Nishiki S, Hasegawa T. 2014. A direct numerical simulation study of vorticity transformation in weakly turbulent premixed flames. Phys. Fluids 26:105104

Lipatnikov AN, Sabelnikov VA. 2013. Transition from countergradient to gradient scalar transport in developing premixed turbulent flames. Flow Turbul. Combust. 90:401-18

Lipatnikov AN, Sabelnikov VA, Nishiki S, Hasegawa T, Chakraborty N. 2015c. DNS assessment of a simple model for evaluating velocity conditioned to unburned gas in premixed turbulent flames. Flow Turbul. Combust. 94:513-26

Majda A, Sethian J. 1985. The derivation and numerical solution of the equations for zero Mach number combustion. Combust. Sci. Technol. 42:185-205

Markstein GH. 1951. Experimental and theoretical studies of flame front stability. F. Aeronaut. Sci. 18:199-220

Matalon M. 2007. Intrinsic flame instabilities in premixed and nonpremixed combustion. Annu. Rev. Fluid Mech. 39:163-91

Moss JB. 1980. Simultaneous measurements of concentration and velocity in an open premixed turbulent flame. Combust. Sci. Techol. 22:119-29

Mura A, Champion M. 2009. Relevance of the Bray number in the small-scale modeling of turbulent premixed flames. Combust. Flame 156:729-33

Nishiki S, Hasegawa T, Borghi R, Himeno R. 2002. Modeling of flame-generated turbulence based on direct numerical simulation databases. Proc. Combust. Inst. 29:2017-22

Nishiki S, Hasegawa T, Borghi R, Himeno R. 2006. Modelling of turbulent scalar flux in turbulent premixed flames based on DNS databases. Combust. Theory Model. 10:39-55 
Nivarti GB, Cant RS. 2015. Aerodynamic quenching and burning velocity of turbulent premixed methane-air flames. Presented at ASME Turbo Expo 2015, Montreal, ASME Pap. GT2015-43416

Paul RN, Bray KNC. 1996. Study of premixed turbulent combustion including Landau-Darrieus instability effects. Proc. Combust. Inst. 26:259-66

Peters N. 2000. Turbulent Combustion. Cambridge, UK: Cambridge Univ. Press

Pfadler S, Leipertz A, Dinkelacker F. 2008. Systematic experiments on turbulent premixed Bunsen flames including turbulent flux measurements. Combust. Flame 152:616-31

Pitsch H. 2006. Large-eddy simulation of turbulent combustion. Annu. Rev. Fluid Mech. 38:453-82

Poinsot T, Veynante D. 2005. Theoretical and Numerical Combustion. Philadelphia: Edwards. 2nd ed.

Poludnenko AY.2015. Pulsating instability and self-acceleration of fast turbulent flames. Phys. Fluids 27:014106

Poludnenko AY, Oran ES. 2010. The interaction of high-speed turbulence with flames: global properties and internal flame structure. Combust. Flame 157:995-1011

Poludnenko AY, Oran ES. 2011. The interaction of high-speed turbulence with flames: turbulent flame speed. Combust. Flame 158:301-26

Prudnikov AG. 1960. Hydrodynamics equations in turbulent flames. In Combustion in a Turbulent Flow, ed. AG Prudnikov, pp. 7-29. Moscow: Oborongiz (In Russian)

Robin V, Mura A, Champion M. 2011. Direct and indirect thermal expansion effects in turbulent premixed flames. F. Fluid Mech. 689:149-82

Robin V, Mura A, Champion M. 2012. Algebraic models for turbulent transports in premixed flames. Combust. Sci. Technol. 184:1718-42

Robin V, Mura A, Champion M, Hasegawa T. 2010. Modeling of the effects of thermal expansion on scalar turbulent fluxes in turbulent premixed flames. Combust. Sci. Tecbnol. 182:449-64

Sabelnikov VA, Lipatnikov AN. 2011. A simple model for evaluating conditioned velocities in premixed turbulent flames. Combust. Sci. Technol. 183:588-613

Sabelnikov VA, Lipatnikov AN. 2013a. Towards an extension of TFC model of premixed turbulent combustion. Flow Turbul. Combust. 90:387-400

Sabelnikov VA, Lipatnikov AN. 2013b. Transition from pulled to pushed premixed turbulent flames due to countergradient transport. Combust. Theory Model. 17:1154-75

Sabelnikov VA, Lipatnikov AN. 2014. Speed selection for traveling-wave solutions to the diffusion-reaction equation with cubic reaction term and Burgers nonlinear convection. Phys. Rev. E 90:033004

Sabelnikov VA, Lipatnikov AN. 2015. Transition from pulled to pushed fronts in premixed turbulent combustion: theoretical and numerical study. Combust. Flame 162:2893-903

Scurlock AC, Grover JH. 1953. Propagation of turbulent flames. Proc. Combust. Inst. 4:645-58

Shchelkin KI. 1967 (1943). On combustion in a turbulent flow. Tech. Memo 1110, Natl. Advis. Comm. Aeronaut., Washington, DC

Shepherd IG, Cheng RK. 2001. The burning rate of premixed flames in moderate and intense turbulence. Combust. Flame 127:2066-75

Sivashinsky GI. 1983. Instabilities, pattern formation, and turbulence in flames. Annu. Rev. Fluid. Mech. 15:179-99

Sponfeldner T, Boxx I, Beyrau F, Hardalupas Y, Meier W, Taylor AMKP. 2015. On the alignment of fluiddynamic principal strain-rates with the $3 \mathrm{D}$ flamelet-normal in a premixed turbulent $\mathrm{V}$-flame. Proc. Combust. Inst. 35:1269-76

Steinberg AM, Coriton B, Frank JH. 2015. Influence of combustion on principal strain-rate transport in turbulent premixed flames. Proc. Combust. Inst. 35:1287-94

Steinberg AM, Driscoll JF. 2009. Straining and wrinkling processes during turbulence-premixed flame interaction measured using temporally-resolved diagnostics. Combust. Flame 156:2285-306

Steinberg AM, Driscoll JF, Ceccio SL. 2009. Temporal evolution of flame stretch due to turbulence and the hydrodynamic instability. Proc. Combust. Inst. 32:1713-21

Steinberg AM, Driscoll JF, Swaminathan N. 2012. Statistics and dynamics of turbulence-flame alignment in premixed combustion. Combust. Flame 159:2576-88

Stevens EJ, Bray KNC, Lecordier B. 1998. Velocity and scalar statistics for premixed turbulent stagnation flames using PIV. Proc. Combust. Inst. 27:949-55 
Swaminathan N, Bray KNC, eds. 2011. Turbulent Premixed Flames. Cambridge, UK: Cambridge Univ. Press Swaminathan N, Grout RW. 2006. Interaction of turbulence and scalar fields in premixed flames. Phys. Fluids 18:045102

Townsend AA. 1976. The Structure of Turbulent Shear Flow. Cambridge, UK: Cambridge Univ. Press. 2nd ed.

Treurniet TC, Nieuwstadt FTM, Boersma BJ. 2006. Direct numerical simulation of homogeneous turbulence in combination with premixed combustion at low Mach number modelled by the G-equation. F. Fluid Mech. 565:25-62

Troiani G, Creta F, Matalon M. 2015. Experimental investigation of Darrieus-Landau instability effects on turbulent premixed flames. Proc. Combust. Inst. 35:1451-59

Troiani G, Marrocco M, Giammartini S, Casciola CM. 2009. Counter-gradient transport in the combustion of a premixed $\mathrm{CH}_{4}$ /air annular jet by combined PIV/OH-LIF. Combust. Flame 156:608-20

Tsinober A. 2009. An Informal Conceptual Introduction to Turbulence. Heidelberg: Springer

Veynante D, Poinsot T. 1997. Effects of pressure gradients on turbulent premixed flames. F. Fluid Mech. 353:83-114

Veynante D, Trouvé A, Bray KNC, Mantel T. 1997. Gradient and counter-gradient scalar transport in turbulent premixed flames. F. Fluid Mech. 332:263-93

Videto BD, Santavicca DA. 1990. Flame-turbulence interactions in a freely-propagating, premixed flame. Combust. Sci. Tecbnol. 70:47-73

Yanagi T, Mimura Y. 1981. Velocity-temperature correlation in premixed flame. Proc. Combust. Inst. 18:103139

Yasari E, Lipatnikov AN. 2015. Assessment of a recent model of turbulent scalar flux in RANS simulations of premixed Bunsen flames. Proc. 8th Int. Symp. Turbul. Heat Mass Transfer. Sarajevo: Begell House $(\mathrm{CD}-\mathrm{ROM})$

Yu R, Bai XS, Bychkov V. 2015a. Fractal flame structure due to the hydrodynamic Darrieus-Landau instability. Phys. Rev. E 92:063028

Yu R, Bay XS, Lipatnikov AN. 2015b. A direct numerical simulation study of interface propagation in homogeneous turbulence. F. Fluid Mech. 772:127-64

Yu R, Lipatnikov AN, Bay XS. 2014. Three-dimensional direct numerical simulation study of conditioned moments associated with front propagation in turbulent flows. Phys. Fluids 26:085104

Zel'dovich YB, Barenblatt GI, Librovich VB, Makhviladze GM. 1985. The Mathematical Theory of Combustion and Explosions. New York: Plenum

Zimont VL, Biagioli F. 2002. Gradient, counter-gradient transport and their transition in turbulent premixed flames. Combust. Theory Model. 6:79-101 